\title{
Existence of an approximate solution for a class of fractional multi-point boundary value problems with the derivative term
}

\section{Yanbin Sang ${ }^{1 *}$ (D) and Luxuan $\mathrm{He}^{1}$}

\section{"Correspondence:}

syb6662004@163.com

'Department of Mathematics,

School of Science, North University

of China, Taiyuan, Shanxi, 030051,

P.R. China

\section{Springer}

\begin{abstract}
In this paper, we consider a class of fractional boundary value problems with the derivative term and nonlinear operator term. By establishing new mixed monotone fixed point theorems, we prove these problems to have a unique solution, and we construct the corresponding iterative sequences to approximate the unique solution.
\end{abstract}

MSC: 65R20; 34B15; 34B18

Keywords: Fractional equation; Mixed monotone operator; Partial order method; Derivative term

\section{Introduction}

In this paper, we consider the existence and uniqueness of nontrivial solutions of the following fractional boundary value problem:

$$
\left\{\begin{array}{l}
D_{0^{+}}^{\beta}\left(D_{0^{+}}^{\alpha} u(t)\right)=f\left(t, u(t), D_{0^{+}}^{v} u(t)\right)+g(t, u(t),(\mathcal{K} u)(t))-b, \quad t \in(0,1), \\
u(0)=u^{\prime}(0)=\cdots=u^{(n-2)}(0)=0 \\
D_{0^{+}}^{\alpha} u(0)=\left(D_{0^{+}}^{\alpha} u\right)^{\prime}(0)=\cdots=\left(D_{0^{+}}^{\alpha} u\right)^{(n-2)}(0)=0 \\
D_{0^{+}}^{\gamma} u(1)=\sum_{i=1}^{m-2} \xi_{i} D_{0^{+}}^{\gamma} u\left(\eta_{i}\right), \quad D_{0^{+}}^{\alpha} u(1)=\sum_{i=1}^{m-2} \zeta_{i} D_{0^{+}}^{\alpha} u\left(\eta_{i}\right),
\end{array}\right.
$$

where $b>0, D_{0^{+}}^{\alpha}, D_{0^{+}}^{\beta}, D_{0^{+}}^{\gamma}, D_{0^{+}}^{\nu}$ are the Riemann-Liouville fractional derivatives with $n-$ $1<\alpha, \beta \leq n, n-2<\gamma \leq n-1, n \geq 2(n \in \mathbb{N}), \alpha-\gamma-1>0,0<v \leq \gamma, 0<\xi_{i}, \eta_{i}, \zeta_{i}<1$, $i=1,2,3, \ldots, m-2, m \geq 2, \sum_{i=1}^{m-2} \xi_{i} \eta_{i}^{\alpha-\gamma-1}<1, \sum_{i=1}^{m-2} \zeta_{i} \eta_{i}^{\beta-1}<1 . f, g:[0,1] \times(-\infty,+\infty) \times$ $(-\infty,+\infty) \longrightarrow(-\infty,+\infty)$ are continuous.

In recent years, much attention has been paid to multi-point boundary value problems involving fractional order; see [1-29] and the references therein. We should mention related studies in [1-22], which motivated us to consider the problem (1.1). Lv [1] studied the existence of positive solutions of the following multi-point boundary value problem:

$$
\left\{\begin{array}{l}
D_{0^{+}}^{\alpha} u(t)+f(t, u(t))=0, \quad t \in(0,1), \\
u(0)=0, \quad D_{0^{+}}^{\beta} u(1)=\sum_{i=1}^{m-2} \xi_{i} D_{0^{+}}^{\beta} u\left(\eta_{i}\right),
\end{array}\right.
$$

(c) The Author(s) 2021. This article is licensed under a Creative Commons Attribution 4.0 International License, which permits use, sharing, adaptation, distribution and reproduction in any medium or format, as long as you give appropriate credit to the original author(s) and the source, provide a link to the Creative Commons licence, and indicate if changes were made. The images or other third party material in this article are included in the article's Creative Commons licence, unless indicated otherwise in a credit line to the material. If material is not included in the article's Creative Commons licence and your intended use is not permitted by statutory regulation or exceeds the permitted use, you will need to obtain permission directly from the copyright holder. To view a copy of this licence, visit http://creativecommons.org/licenses/by/4.0/. 
where $1<\alpha \leq 2,0 \leq \beta \leq 1,0 \leq \alpha-\beta-1,0<\xi_{i}, \eta_{i}<1(i=1,2, \ldots, m-2)$, and $\sum_{i=1}^{m-2} \xi_{i} \eta_{i}^{\alpha-\beta-1}<1 . f:[0,1] \times[0,+\infty) \longrightarrow[0,+\infty)$ is a continuous function. Lv [1] first obtained the Green's function of linear boundary value problem corresponding to the problem (1.2), which has been adopted in the proof of main theorems in [2-4]. Furthermore, $\mathrm{Lv}[2]$ studied the existence of solutions for nonlinear fractional $m$-point boundary value problems involving $p$-Laplacian operators by the fixed point index theorem. $\mathrm{Li}$, Luo and Zhou [5] discussed Eq. (1.2) in the case of $m=3$ by using some fixed point theorems.

Very recently, Wang, Zhang and Wang [6] considered the following nonlinear fractional boundary value problem:

$$
\left\{\begin{array}{l}
-D_{0^{+}}^{\alpha} u(t)=f(t, u(t), u(t))+g(t, u(t)), \quad 0<t<1, n-1<\alpha \leq n, \\
u^{(i)}(0)=0, \quad i=0, \ldots, n-2, \quad D_{0^{+}}^{v} u(1)=b D_{0^{+}}^{v} u(\xi), \quad n-2<v \leq n-1,
\end{array}\right.
$$

where $n-1<\alpha \leq n(n>2, n \in \mathbb{N}), n-2<v \leq n-1,0 \leq b \leq 1,0<\xi<1$ satisfying $\alpha-$ $v-1 \geq 0$ and $0 \leq b \xi^{\alpha-v-1}<1$. They established the existence and uniqueness of solutions of (1.3) by applying the properties of Green function and fixed point theorems for sumtype operator. On the other hand, they also gave the physical application of our system (1.1). The main feature of [6] is that the value of $\alpha$ is extended from $1<\alpha \leq 2$ in (1.2) to $n-1<\alpha \leq n$. Liang and Zhang [7] considered the existence of solutions of the problem (1.3) when $n=4, v=2$ and $f(t, u(t), u(t))=0$. Moreover, Jleli and Samet [8] gave some sufficient conditions under which the problem (1.3) has a unique positive solution when $b=0$.

We note that Wang [9] studied the existence and uniqueness of positive solutions for singular fractional differential equations as follows:

$$
\left\{\begin{array}{l}
D_{0^{+}}^{\alpha} u(t)+p(t) f\left(t, u(t), D_{0^{+}}^{\beta} u(t)\right)+q(t) g(t, u(t),(H u)(t))=0, \quad 0<t<1, \\
u(0)=u^{\prime}(0)=\cdots=u^{(n-2)}(0)=0, \quad\left[D_{0^{+}}^{\gamma} u(t)\right]_{t=1}=k(u(1)),
\end{array}\right.
$$

where $n-1<\alpha \leq n, n>3,1 \leq \beta \leq \gamma \leq n-2, p, q \in C((0,1),[0,+\infty)), p(t)$ and $q(t)$ are allowed to be singular at $t=0$ or $t=1$. $f, g:(0,1) \times[0,+\infty) \times[0,+\infty) \longrightarrow[0,+\infty)$ are continuous, and $k:[0,1) \longrightarrow[0,+\infty)$ is also continuous. What attracts our attention is the nonlinear term contains not only the derivative term but also the operator term $(\mathrm{Hu})$. Similarly, Zhang and Tian [10] also studied the problem (1.4) with derivative term, but the difference is that the function $g$ does not include the operator term. In [11], Ji et al. also investigated positive solutions for the nonlinear fractional differential equation with a derivative term. Goodrich [12] first obtained the Green function of the problem (1.4) when $k(u(1))=0$. In [13-15], they considered the fractional differential equations with integer order derivative, and they did not consider the boundary condition $\left[D_{0^{+}}^{\gamma} u(t)\right]_{t=1}$. Zhang [16] considered the singular fractional differential equations with multiple derivative terms, and obtained the existence of positive solutions.

We should mention the work of Jong [3], which directly is related to our problem (1.1). Jong investigated the following nonlinear fractional $m$-point boundary value problem with 
$p$-Laplacian operator:

$$
\left\{\begin{array}{l}
D_{0^{+}}^{\beta}\left(\varphi_{p}\left(D_{0^{+}}^{\alpha} u\right)\right)(t)=f(t, u(t)), \quad t \in(0,1), \\
u(0)=0, \quad D_{0^{+}}^{\alpha} u(0)=0, \\
D_{0^{+}}^{\gamma} u(1)=\sum_{i=1}^{m-2} \xi_{i} D_{0^{+}}^{\gamma} u\left(\eta_{i}\right), \quad \varphi_{p}\left(D_{0^{+}}^{\alpha} u\right)(1)=\sum_{i=1}^{m-2} \zeta_{i} \varphi_{p}\left(D_{0^{+}}^{\alpha} u\right)\left(\eta_{i}\right),
\end{array}\right.
$$

where $1<\alpha, \beta \leq 2,3<\alpha+\beta \leq 4,0<\gamma \leq 1, \alpha-\gamma-1>0,0<\xi_{i}, \eta_{i}, \zeta_{i}<1, \sum_{i=1}^{m-2} \xi_{i} \eta_{i}^{\alpha-\gamma-1}<1$, $\sum_{i=1}^{m-2} \zeta_{i} \eta_{i}^{\beta-1}<1$, the $p$-Laplacian operator is defined as $\varphi_{p}(s)=|s|^{p-2} s, p>1$. Jong obtained that the problem (1.5) has a unique solution which is given by $u(t)=\int_{0}^{1} G(t, s) \times$ $\varphi_{p}^{-1}\left(\int_{0}^{1} H(s, \tau) f(\tau, u(\tau)) d \tau\right) d s$. He first gave the Green function $H(s, \tau)$. The main tool of [3] is the Banach contraction mapping principle. Furthermore, he also showed the uniqueness of the problem (1.5) in [4] by the classic fixed point theorem of mixed monotone operators. Li and Qi [17] focused on $p$-Laplacian boundary value problems of higher order nonlinear differential equations. Tan and Li [18] used Kuratowski's noncompactness measure and Sadovskii's fixed point theorem to study the problem (1.5) when the boundary condition $\varphi_{p}\left(D_{0^{+}}^{\alpha} u\right)(1)=\sum_{i=1}^{m-2} \zeta_{i} \varphi_{p}\left(D_{0^{+}}^{\alpha} u\right)\left(\eta_{i}\right)$ is removed. Wang and Xiang [19] considered the problem (1.5) when all boundary conditions are replaced by $u(0)=0, D_{0^{+}}^{\alpha} u(0)=0$, $u(1)=a u(\xi)$ and $D_{0^{+}}^{v} u(1)=b D_{0^{+}}^{\alpha} u(\eta)$. Wang, Xiang and Liu [20] investigated the problem (1.5) when the boundary conditions are replaced by $u(0)=0, D_{0^{+}}^{\alpha} u(0)=0$ and $u(1)=a u(\xi)$. We should point out that the main tools and methods adopted in [1-20] are cone mapping theory. Therefore, nonlinearities in the problems (1.2)-(1.5) are usually required to be non-negative. But more and more authors are beginning to remove this restriction imposed on nonlinear terms. Very recently, Sang and Ren [21] dealt with the following fractional boundary value problem:

$$
\left\{\begin{array}{l}
-D_{0^{+}}^{\alpha} u(t)=f(t, u(t), u(t))+g(t, u(t), u(t))-b, \quad 0<t<1 \\
u^{(i)}(0)=0, \quad 0 \leq i \leq n-2, \quad\left[D_{0^{+}}^{\beta} u(t)\right]_{t=1}=0
\end{array}\right.
$$

where $n-1<\alpha \leq n, 1 \leq \beta \leq n-2, n \geq 3(n \in \mathbb{N}), b>0$ is a constant, $f, g:[0,1] \times$ $(-\infty,+\infty) \times(-\infty,+\infty) \longrightarrow(-\infty,+\infty)$ are two continuous functions. In fact, Zhai and Wang [22] have introduced $\varphi-(h, e)$ operators, and established the existence and uniqueness of a nontrivial solution for a class of nonlinear fractional equations by using partial order method.

In this paper, the first goal is to obtain the fixed point of the solution of the operator equation $M(x, x)+N(x, x)+e=x$, where $M$ and $N$ are two mixed monotone operators. We will generalize the results of cone mapping to the non-cone case. Then we will provide some sufficient conditions under which the problem (1.1) has a unique solution and construct two iterative sequences of unique solution. Compared with [6,9], we do not demand the assumption that nonlinearities are non-negative, and the more general boundary conditions are adopted.

Our paper is organized as follows. In Sect. 2, we will introduce some definitions and give some lemmas to prove the main conclusions. In Sect. 3, the existence of fixed point for the operator equation associated with the problem (1.1) is established. Then, based on our abstract results, the existence and uniqueness of the solution of the problem (1.1) are proved. 


\section{Preliminaries and related lemmas}

In this section, we give some definitions and lemmas that are useful for the proof of our main results.

In this paper, $(E,\|\cdot\|)$ is a real Banach space. A partially ordered structure in $E$ is induced by a cone $P \subset E$, i.e. $x \leq y$ if and only if $y-x \in P$. $\theta$ is the zero element. $P$ is called normal if there exists $N>0$ such that $\theta \leq x \leq y \Rightarrow\|x\| \leq N\|y\|$. Given $h>\theta$, we denote by $P_{h}$

$$
P_{h}=\{x \in E \mid \text { there exist } \lambda>0, \mu>0 \text { such that } \lambda h \leq x \leq \mu h\} .
$$

Let $e \in P$ with $\theta \leq e \leq h$, denote

$$
P_{h, e}=\left\{x \in E \mid x+e \in P_{h}\right\} .
$$

Definition 2.1 ([30,31]) If $A(x, y)$ is increasing in $x$, and decreasing in $y$, then $A: P_{h, e} \times$ $P_{h, e} \rightarrow E$ is a mixed monotone operator. That is, for every $u_{i}, v_{i} \in P_{h, e}(i=1,2)$ with $u_{1} \geq v_{1}$, $u_{2} \leq v_{2}$, we have $A\left(u_{1}, u_{2}\right) \geq A\left(v_{1}, v_{2}\right)$.

Definition 2.2 ([32,33]) The Riemann-Liouville fractional derivative of order $\alpha>0$ of a function $h \in C[0,1]$ is defined by

$$
D_{0^{+}}^{\alpha} h(t)=\frac{1}{\Gamma(n-\alpha)}\left(\frac{d}{d t}\right)^{n} \int_{0}^{t} h(s)(t-s)^{n-\alpha-1} d s,
$$

where $n=[\alpha]+1$. The Riemann-Liouville fractional integral of order $\alpha>0$ of a function $h$ is given by

$$
I_{0^{+}}^{\alpha} h(t)=\frac{1}{\Gamma(\alpha)} \int_{0}^{t}(t-s)^{\alpha-1} h(s) d s .
$$

Definition 2.3 ([32]) Let $\alpha>-1, v>0$ and $t>0$. Then

$$
D_{0^{+}}^{v} t^{\alpha}=\frac{\Gamma(\alpha+1)}{\Gamma(\alpha-v+1)} t^{\alpha-v}
$$

Lemma 2.1 ([34]) Let $u \in C[0,1] \cap L^{1}[0,1], \alpha>0$, then

$$
I_{0^{+}}^{\alpha} D_{0^{+}}^{\alpha} u(t)=u(t)+c_{1} t^{\alpha-1}+c_{2} t^{\alpha-2}+\cdots+c_{n} t^{\alpha-n}
$$

where $c_{i} \in \mathbb{R}, i=1,2, \ldots, n$ and $n=[\alpha]+1$.

Lemma 2.2 Let $\sum_{i=1}^{m-2} \xi_{i} \eta_{i}^{\alpha-\gamma-1} \neq 1$. If $y(t) \in C[0,1]$, then the boundary value problem

$$
\left\{\begin{array}{l}
D_{0^{+}}^{\alpha} u(t)+y(t)=0, \quad 0<t<1, n-1<\alpha \leq n, \\
u(0)=u^{\prime}(0)=\cdots=u^{(n-2)}(0)=0, \\
D_{0^{+}}^{\gamma} u(1)=\sum_{i=1}^{m-2} \xi_{i} D_{0^{+}}^{\gamma} u\left(\eta_{i}\right), \quad n-2<\gamma \leq n-1,
\end{array}\right.
$$

has a unique solution

$$
u(t)=\int_{0}^{1} G(t, s) y(s) d s
$$


where

$$
\begin{aligned}
& G(t, s)=G_{1}(t, s)+G_{2}(t, s), \\
& G_{1}(t, s)=\frac{1}{\Gamma(\alpha)} \begin{cases}t^{\alpha-1}(1-s)^{\alpha-\gamma-1}-(t-s)^{\alpha-1}, & 0 \leq s \leq t \leq 1, \\
t^{\alpha-1}(1-s)^{\alpha-\gamma-1}, & 0 \leq t \leq s \leq 1,\end{cases}
\end{aligned}
$$

and

$$
G_{2}(t, s)=\frac{1}{A \Gamma(\alpha)} \begin{cases}t^{\alpha-1} \sum_{0 \leq s \leq \eta_{i}} \xi_{i}\left[\eta_{i}^{\alpha-\gamma-1}(1-s)^{\alpha-\gamma-1}-\left(\eta_{i}-s\right)^{\alpha-\gamma-1}\right], & 0 \leq t \leq 1 \\ t^{\alpha-1} \sum_{\eta_{i} \leq s \leq 1} \xi_{i} \eta_{i}^{\alpha-\gamma-1}(1-s)^{\alpha-\gamma-1}, & 0 \leq t \leq 1\end{cases}
$$

with

$$
A=1-\sum_{i=1}^{m-2} \xi_{i} \eta_{i}^{\alpha-\gamma-1}
$$

Proof Using Lemma 2.1, we get

$$
u(t)+c_{1} t^{\alpha-1}+c_{2} t^{\alpha-2}+\cdots+c_{n} t^{\alpha-n}=-I_{0^{+}}^{\alpha} y(t) .
$$

It follows from the condition $u(0)=u^{\prime}(0)=\cdots=u^{(n-2)}(0)=0$ that $c_{n}=c_{n-1}=\cdots=c_{2}=0$.

Thus

$$
u(t)=-I_{0^{+}}^{\alpha} y(t)-c_{1} t^{\alpha-1} .
$$

The rest of our proof can be obtained from Lemma 2.1 in [1].

Lemma 2.3 Let $\sum_{i=1}^{m-2} \zeta_{i} \eta_{i}^{\beta-1} \neq 1$. Iff : $[0,1] \times(-\infty,+\infty) \times(-\infty,+\infty) \rightarrow(-\infty,+\infty)$ be a continuous function. Then the problem (1.1) has the following unique solution:

$$
u(t)=\int_{0}^{1} G(t, s)\left(\int_{0}^{1} H(s, \tau)\left(f\left(\tau, u(\tau), D_{0^{+}}^{v} u(\tau)\right)+g(\tau, u(\tau),(\mathcal{K} u)(\tau))-b\right) d \tau\right) d s
$$

where

$$
H(t, s)=H_{1}(t, s)+H_{2}(t, s),
$$

in which

$$
\begin{aligned}
& H_{1}(t, s)=\frac{1}{\Gamma(\beta)} \begin{cases}t^{\beta-1}(1-s)^{\beta-1}-(t-s)^{\beta-1}, & 0 \leq s \leq t \leq 1, \\
t^{\beta-1}(1-s)^{\beta-1}, & 0 \leq t \leq s \leq 1,\end{cases} \\
& H_{2}(t, s)=\frac{1}{B \Gamma(\beta)} \begin{cases}t^{\beta-1} \sum_{0 \leq s \leq \eta_{i}} \zeta_{i}\left[\eta_{i}^{\beta-1}(1-s)^{\beta-1}-\left(\eta_{i}-s\right)^{\beta-1}\right], & 0 \leq t \leq 1, \\
t^{\beta-1} \sum_{\eta_{i} \leq s \leq 1} \zeta_{i} \eta_{i}^{\beta-1}(1-s)^{\beta-1}, & 0 \leq t \leq 1,\end{cases}
\end{aligned}
$$


where

$$
B=1-\sum_{i=1}^{m-2} \zeta_{i} \eta_{i}^{\beta-1}
$$

Proof Let $h \in C[0,1]$, consider the boundary value problem:

$$
\left\{\begin{array}{l}
D_{0^{+}}^{\beta} \nu(t)+h(t)=0, \quad 0<t<1, n-1<\beta \leq n, \\
v(0)=v^{\prime}(0)=\cdots=v^{(n-2)}(0)=0, \quad v(1)=\sum_{i=1}^{m-2} \zeta_{i} v\left(\eta_{i}\right) .
\end{array}\right.
$$

Similarly, using Lemma 2.1, we deduce

$$
v(t)+c_{1} t^{\beta-1}+c_{2} t^{\beta-2}+\cdots+c_{n} t^{\beta-n}=-I_{0^{+}}^{\beta} h(t) .
$$

It follows from the condition $v(0)=v^{\prime}(0)=\cdots=v^{(n-2)}(0)=0$ that $c_{n}=c_{n-1}=\cdots=c_{2}=0$. Thus

$$
v(t)=-I_{0^{+}}^{\beta} h(t)-c_{1} t^{\beta-1} .
$$

The rest of our proof can be derived from Lemma 2.4 in [3].

Lemma 2.4 Let

$$
\begin{aligned}
& C(s)=\frac{1}{A} \sum_{0 \leq s \leq \eta_{i}} \xi_{i}\left[\eta_{i}^{\alpha-\gamma-1}(1-s)^{\alpha-\gamma-1}-\left(\eta_{i}-s\right)^{\alpha-\gamma-1}\right]+\sum_{s \geq \eta_{i}} \xi_{i} \eta_{i}^{\alpha-\gamma-1}(1-s)^{\alpha-\gamma-1}, \\
& D=\frac{1}{A}\left(1+\sum_{i=1}^{m-2} \xi_{i}\left(1-\eta_{i}^{\alpha-\gamma-1}\right)\right) .
\end{aligned}
$$

Then the function $G(t, s)$ defined in Lemma 2.2 satisfies the following conditions:

$$
C(s) t^{\alpha-1} \leq \Gamma(\alpha) G(t, s) \leq D t^{\alpha-1},
$$

and

$$
C(s) t^{\alpha-v-1} \leq \Gamma(\alpha-v) D_{0^{+}}^{v} G(t, s) \leq D t^{\alpha-v-1},
$$

for every $t, s \in[0,1]$.

Proof Since $G_{1}(t, s) \geq 0$ for $t \in[0,1], s \in[0,1]$, it follows that

$$
G(t, s) \geq G_{2}(t, s)=\frac{C(s)}{\Gamma(\alpha)} t^{\alpha-1} .
$$


At the same time, we have

$$
\begin{aligned}
G(t, s) & =G_{1}(t, s)+G_{2}(t, s) \\
& \leq \frac{1}{\Gamma(\alpha)} t^{\alpha-1}+\frac{1}{A \Gamma(\alpha)} \sum_{i=1}^{m-2} \xi_{i} t^{\alpha-1} \\
& =\frac{1}{A \Gamma(\alpha)}\left(1+\sum_{i=1}^{m-2} \xi_{i}\left(1-\eta_{i}^{\alpha-\gamma-1}\right)\right) t^{\alpha-1} \\
& =\frac{D}{\Gamma(\alpha)} t^{\alpha-1} .
\end{aligned}
$$

Consequently

$$
C(s) t^{\alpha-1} \leq \Gamma(\alpha) G(t, s) \leq D t^{\alpha-1} .
$$

Similarly

$$
D_{0^{+}}^{v} G(t, s) \geq D_{0^{+}}^{v} G_{2}(t, s)=\frac{C(s)}{\Gamma(\alpha-v)} t^{\alpha-v-1}
$$

and

$$
\begin{aligned}
D_{0^{+}}^{v} G(t, s) & =D_{0^{+}}^{v} G_{1}(t, s)+D_{0^{+}}^{v} G_{2}(t, s) \\
& \leq \frac{1}{\Gamma(\alpha-v)} t^{\alpha-v-1}+\frac{1}{A \Gamma(\alpha-v)} \sum_{i=1}^{m-2} \xi_{i} t^{\alpha-v-1} \\
& =\frac{1}{A \Gamma(\alpha-v)}\left(1+\sum_{i=1}^{m-2} \xi_{i}\left(1-\eta_{i}^{\alpha-\gamma-1}\right)\right) t^{\alpha-v-1} \\
& =\frac{D}{\Gamma(\alpha-v)} t^{\alpha-v-1} .
\end{aligned}
$$

Hence

$$
C(s) t^{\alpha-v-1} \leq \Gamma(\alpha-v) D_{0^{+}}^{v} G(t, s) \leq D t^{\alpha-v-1}
$$

Lemma 2.5 ([4]) Let

$$
E(s)=\frac{1}{B} \sum_{0 \leq s \leq \eta_{i}} \zeta_{i}\left[\eta_{i}^{\beta-1}(1-s)^{\beta-1}-\left(\eta_{i}-s\right)^{\beta-1}\right]+\sum_{s \geq \eta_{i}} \zeta_{i} \eta_{i}^{\beta-1}(1-s)^{\beta-1}
$$

and

$$
F=\frac{1}{B}\left(1+\sum_{i=1}^{m-2} \zeta_{i}\left(1-\eta_{i}^{\beta-1}\right)\right)
$$

Then

$$
E(s) t^{\beta-1} \leq \Gamma(\beta) H(t, s) \leq F t^{\beta-1},
$$

for every $t, s \in[0,1]$. 
Lemma 2.6 Let P be a normal cone and $T: P_{h, e} \times P_{h, e} \longrightarrow$ E be a mixed monotone operator. Assume that the following conditions hold:

(i) for every $\lambda \in(0,1)$ and $u, v \in P_{h, e}$, there exists $\varphi(\lambda, u, v)>\lambda$ such that

$$
T\left(\lambda u+(\lambda-1) e, \lambda^{-1} v+\left(\lambda^{-1}-1\right) e\right) \geq \varphi(\lambda, u, v) T(u, v)+(\varphi(\lambda, u, v)-1) e ;
$$

(ii) for fixed $t \in(0,1)$ and $u \in P_{h, e}, \varphi(t, u, v)$ is decreasing in $v$, and for fixed $t \in(0,1)$ and $v \in P_{h, e}, \varphi(t, u, v)$ is increasing in $u$;

(iii) there exists $t_{0} \in(0,1)$ such that

$$
\frac{t_{0}}{\varphi\left(t_{0}, h, h\right)} h+\left(\frac{t_{0}}{\varphi\left(t_{0}, h, h\right)}-1\right) e \leq T(h, h) \leq \frac{1}{t_{0}} h+\left(\frac{1}{t_{0}}-1\right) e .
$$

Then:

(1) $T$ has a unique fixed point $x^{*}$ in $P_{h, e}$;

(2) there exist initial values $u_{0}, v_{0} \in P_{h, e}$, and $s \in(0,1)$ such that

$$
s v_{0} \leq u_{0}<v_{0}, \quad u_{0} \leq T\left(u_{0}, v_{0}\right) \leq T\left(v_{0}, u_{0}\right) \leq v_{0} ;
$$

(3) for any $x_{0}, y_{0} \in P_{h, e}$, taking the iterative sequences as follows:

$$
x_{n}=T\left(x_{n-1}, y_{n-1}\right), \quad y_{n}=T\left(y_{n-1}, x_{n-1}\right), \quad n=1,2, \ldots
$$

we have $x_{n} \rightarrow x^{*}$ and $y_{n} \rightarrow x^{*}$ as $n \rightarrow \infty$.

Proof By (i), we have

$$
\begin{aligned}
& T\left(\lambda^{-1} u+\left(\lambda^{-1}-1\right) e, \lambda v+(\lambda-1) e\right) \\
& \leq {\left[\varphi\left(\lambda, \lambda^{-1} u+\left(\lambda^{-1}-1\right) e, \lambda v+(\lambda-1) e\right)\right]^{-1} T(u, v) } \\
&+\left(\left[\varphi\left(\lambda, \lambda^{-1} u+\left(\lambda^{-1}-1\right) e, \lambda v+(\lambda-1) e\right)\right]^{-1}-1\right) e
\end{aligned}
$$

for every $\lambda \in(0,1), u, v \in P_{h, e}$. We can find a positive integer $k$ with

$$
\left(\frac{\varphi\left(t_{0}, h, h\right)}{t_{0}}\right)^{k} \geq \frac{1}{t_{0}}
$$

Let

$$
\begin{aligned}
& u_{n}=T\left(u_{n-1}, v_{n-1}\right), \quad v_{n}=T\left(v_{n-1}, u_{n-1}\right), \quad n=1,2, \ldots \\
& x_{n}=t_{0}^{n} h+\left(t_{0}^{n}-1\right) e, \quad y_{n}=t_{0}^{-n} h+\left(t_{0}^{-n}-1\right) e, \quad n=1,2, \ldots
\end{aligned}
$$

Thus

$$
x_{n}=t_{0} x_{n-1}+\left(t_{0}-1\right) e, \quad y_{n}=t_{0}^{-1} y_{n-1}+\left(t_{0}^{-1}-1\right) e, \quad n=1,2, \ldots
$$

Denote $u_{0}:=x_{k}, v_{0}:=y_{k}$, then $u_{0}, v_{0} \in P_{h, e}$,

$$
u_{0} \leq v_{0}, \quad u_{1}=T\left(u_{0}, v_{0}\right) \leq T\left(v_{0}, u_{0}\right)=v_{1} .
$$


Since $T$ is mixed monotone, we get

$$
u_{n} \leq v_{n}, \quad n=1,2, \ldots
$$

By the conditions (ii) and (iii), combining with (2.1), we have

$$
\begin{aligned}
& u_{1}=T\left(u_{0}, v_{0}\right) \\
& =T\left(t_{0}^{k} h+\left(t_{0}^{k}-1\right) e, t_{0}^{-k} h+\left(t_{0}^{-k}-1\right) e\right) \\
& =T\left(t_{0}\left(t_{0}^{k-1} h+\left(t_{0}^{k-1}-1\right) e\right)+\left(t_{0}-1\right) e, t_{0}^{-1}\left(t_{0}^{-k+1} h+\left(t_{0}^{-k+1}-1\right) e\right)+\left(t_{0}^{-1}-1\right) e\right) \\
& \geq \varphi\left(t_{0}, t_{0}^{k-1} h+\left(t_{0}^{k-1}-1\right) e, t_{0}^{-k+1} h+\left(t_{0}^{-k+1}-1\right) e\right) T\left(t_{0}^{k-1} h+\left(t_{0}^{k-1}-1\right) e, t_{0}^{-k+1} h\right. \\
& \left.+\left(t_{0}^{-k+1}-1\right) e\right)+\left(\varphi\left(t_{0}, t_{0}^{k-1} h+\left(t_{0}^{k-1}-1\right) e, t_{0}^{-k+1} h+\left(t_{0}^{-k+1}-1\right) e\right)-1\right) e \\
& \geq \varphi\left(t_{0}, t_{0}^{k-1} h+\left(t_{0}^{k-1}-1\right) e, t_{0}^{-k+1} h+\left(t_{0}^{-k+1}-1\right) e\right)\left[\varphi \left(t_{0}, t_{0}^{k-2} h+\left(t_{0}^{k-2}-1\right) e,\right.\right. \\
& \left.t_{0}^{-k+2} h+\left(t_{0}^{-k+2}-1\right) e\right) T\left(t_{0}^{k-2} h+\left(t_{0}^{k-2}-1\right) e, t_{0}^{-k+2} h+\left(t_{0}^{-k+2}-1\right) e\right) \\
& \left.+\left(\varphi\left(t_{0}, t_{0}^{k-2} h+\left(t_{0}^{k-2}-1\right) e, t_{0}^{-k+2} h+\left(t_{0}^{-k+2}-1\right) e\right)-1\right) e\right] \\
& +\left(\varphi\left(t_{0}, t_{0}^{k-1} h+\left(t_{0}^{k-1}-1\right) e, t_{0}^{-k+1} h+\left(t_{0}^{-k+1}-1\right) e\right)-1\right) e \\
& =\varphi\left(t_{0}, t_{0}^{k-1} h+\left(t_{0}^{k-1}-1\right) e, t_{0}^{-k+1} h+\left(t_{0}^{-k+1}-1\right) e\right) \varphi\left(t_{0}, t_{0}^{k-2} h+\left(t_{0}^{k-2}-1\right) e, t_{0}^{-k+2} h\right. \\
& \left.+\left(t_{0}^{-k+2}-1\right) e\right) T\left(t_{0}^{k-2} h+\left(t_{0}^{k-2}-1\right) e, t_{0}^{-k+2} h+\left(t_{0}^{-k+2}-1\right) e\right)+\left[\varphi \left(t_{0}, t_{0}^{k-1} h\right.\right. \\
& \left.+\left(t_{0}^{k-1}-1\right) e, t_{0}^{-k+1} h+\left(t_{0}^{-k+1}-1\right) e\right) \varphi\left(t_{0}, t_{0}^{k-2} h+\left(t_{0}^{k-2}-1\right) e, t_{0}^{-k+2} h\right. \\
& \left.\left.+\left(t_{0}^{-k+2}-1\right) e\right)-1\right] e \\
& \geq \cdots \geq \varphi\left(t_{0}, t_{0}^{k-1} h+\left(t_{0}^{k-1}-1\right) e, t_{0}^{-k+1} h+\left(t_{0}^{-k+1}-1\right) e\right) \varphi\left(t_{0}, t_{0}^{k-2} h+\left(t_{0}^{k-2}-1\right) e,\right. \\
& \left.t_{0}^{-k+2} h+\left(t_{0}^{-k+2}-1\right) e\right) \ldots \varphi\left(t_{0}, h, h\right) T(h, h)+\left[\varphi \left(t_{0}, t_{0}^{k-1} h+\left(t_{0}^{k-1}-1\right) e, t_{0}^{-k+1} h\right.\right. \\
& \left.\left.+\left(t_{0}^{-k+1}-1\right) e\right) \varphi\left(t_{0}, t_{0}^{k-2} h+\left(t_{0}^{k-2}-1\right) e, t_{0}^{-k+2} h+\left(t_{0}^{-k+2}-1\right) e\right) \cdots \varphi\left(t_{0}, h, h\right)-1\right] e \\
& \geq t_{0}^{k-1} \varphi\left(t_{0}, h, h\right) T(h, h)+\left(t_{0}^{k-1} \varphi\left(t_{0}, h, h\right)-1\right) e \\
& \geq t_{0}^{k-1} \varphi\left(t_{0}, h, h\right)\left(\frac{t_{0}}{\varphi\left(t_{0}, h, h\right)} h+\left(\frac{t_{0}}{\varphi\left(t_{0}, h, h\right)}-1\right) e\right)+\left(t_{0}^{k-1} \varphi\left(t_{0}, h, h\right)-1\right) e \\
& =t_{0}^{k} h+\left(t_{0}^{k}-t_{0}^{k-1} \varphi\left(t_{0}, h, h\right)\right) e+\left(t_{0}^{k-1} \varphi\left(t_{0}, h, h\right)-1\right) e \\
& =t_{0}^{k} h+\left(t_{0}^{k}-1\right) e=x_{k}=u_{0}
\end{aligned}
$$

and

$$
\begin{aligned}
v_{1}= & T\left(v_{0}, u_{0}\right) \\
= & T\left(t_{0}^{-k} h+\left(t_{0}^{-k}-1\right) e, t_{0}^{k} h+\left(t_{0}^{k}-1\right) e\right) \\
\leq & \varphi\left(t_{0}, t_{0}^{-k} h+\left(t_{0}^{-k}-1\right) e, t_{0}^{k} h+\left(t_{0}^{k}-1\right) e\right)^{-1} T\left(t_{0}^{-k+1} h+\left(t_{0}^{-k+1}-1\right) e, t_{0}^{k-1} h\right. \\
& \left.+\left(t_{0}^{k-1}-1\right) e\right)+\left(\varphi\left(t_{0}, t_{0}^{-k} h+\left(t_{0}^{-k}-1\right) e, t_{0}^{k} h+\left(t_{0}^{k}-1\right) e\right)^{-1}-1\right) e \\
\leq & \varphi\left(t_{0}, t_{0}^{-k} h+\left(t_{0}^{-k}-1\right) e, t_{0}^{k} h+\left(t_{0}^{k}-1\right) e\right)^{-1} \varphi\left(t_{0}, t_{0}^{-k+1} h+\left(t_{0}^{-k+1}-1\right) e, t_{0}^{k-1} h\right. \\
& \left.+\left(t_{0}^{k-1}-1\right) e\right)^{-1} T\left(t_{0}^{-k+1} h+\left(t_{0}^{-k+1}-1\right) e, t_{0}^{k-1} h+\left(t_{0}^{k-1}-1\right) e\right)+\left[\varphi \left(t_{0}, t_{0}^{-k} h\right.\right.
\end{aligned}
$$




$$
\begin{aligned}
& \left.+\left(t_{0}^{-k}-1\right) e, t_{0}^{k} h+\left(t_{0}^{k}-1\right) e\right)^{-1} \varphi\left(t_{0}, t_{0}^{-k+1} h+\left(t_{0}^{-k+1}-1\right) e, t_{0}^{k-1} h\right. \\
& \left.\left.+\left(t_{0}^{k-1}-1\right) e\right)^{-1}-1\right] e \\
\leq & \cdots \leq \varphi\left(t_{0}, t_{0}^{-k} h+\left(t_{0}^{-k}-1\right) e, t_{0}^{k} h+\left(t_{0}^{k}-1\right) e\right)^{-1} \varphi\left(t_{0}, t_{0}^{-k+1} h+\left(t_{0}^{-k+1}-1\right) e,\right. \\
& \left.t_{0}^{k-1} h+\left(t_{0}^{k-1}-1\right) e\right)^{-1} \cdots \varphi\left(t_{0}, t_{0}^{-1} h+\left(t_{0}^{-1}-1\right) e, t_{0} h+\left(t_{0}-1\right) e\right)^{-1} T(h, h) \\
& +\left[\varphi ( t _ { 0 } , t _ { 0 } ^ { - k } h + ( t _ { 0 } ^ { - k } - 1 ) e , t _ { 0 } ^ { k } h + ( t _ { 0 } ^ { k } - 1 ) e ) ^ { - 1 } \varphi \left(t_{0}, t_{0}^{-k+1} h+\left(t_{0}^{-k+1}-1\right) e, t_{0}^{k-1} h\right.\right. \\
& \left.\left.+\left(t_{0}^{k-1}-1\right) e\right)^{-1} \cdots \varphi\left(t_{0}, t_{0}^{-1} h+\left(t_{0}^{-1}-1\right) e, t_{0} h+\left(t_{0}-1\right) e\right)^{-1}-1\right] e \\
\leq & \varphi\left(t_{0}, t_{0}^{-1} h+\left(t_{0}^{-1}-1\right) e, t_{0} h+\left(t_{0}-1\right) e\right)^{-k} T(h, h) \\
& +\left[\varphi\left(t_{0}, t_{0}^{-1} h+\left(t_{0}^{-1}-1\right) e, t_{0} h+\left(t_{0}-1\right) e\right)^{-k}-1\right] e \\
\leq & \varphi\left(t_{0}, t_{0}^{-1} h, t_{0} h\right)^{-k} T(h, h)+\left[\varphi\left(t_{0}, t_{0}^{-1} h, t_{0} h\right)^{-k}-1\right] e \\
\leq & \varphi\left(t_{0}, h, h\right)^{-k} T(h, h)+\left[\varphi\left(t_{0}, h, h\right)^{-k}-1\right] e \\
\leq & \varphi\left(t_{0}, h, h\right)^{-k}\left[t_{0}^{-1} h+\left(t_{0}^{-1}-1\right) e\right]+\left[\varphi\left(t_{0}, h, h\right)^{-k}-1\right] e \\
= & \varphi\left(t_{0}, h, h\right)^{-k} t_{0}^{-1} h+\varphi\left(t_{0}, h, h\right)^{-k} t_{0}^{-1} e-e \\
\leq & t_{0}^{-k} h+\left(t_{0}^{-k}-1\right) e=y_{k}=v_{0} .
\end{aligned}
$$

Thus

$$
u_{0} \leq T\left(u_{0}, v_{0}\right) \leq T\left(v_{0}, u_{0}\right) \leq v_{0} .
$$

We deduce for all $n \in \mathbb{N}$ that

$$
u_{0} \leq u_{1} \leq \cdots \leq u_{n} \leq \cdots \leq v_{n} \leq \cdots \leq v_{1} \leq v_{0} .
$$

In addition

$$
u_{n} \geq u_{0} \geq s v_{0}+(s-1) e \geq s v_{n}+(s-1) e, \quad n=1,2, \ldots
$$

Let

$$
t_{n}=\sup \left\{t>0 \mid u_{n} \geq t v_{n}+(t-1) e\right\} .
$$

Thus we have $u_{n} \geq t_{n} v_{n}+\left(t_{n}-1\right) e, n=1,2, \ldots$. Consequently $\left\{t_{n}\right\}$ is increasing with $\left\{t_{n}\right\} \subset$ $(0,1]$. Assume that $t_{n} \rightarrow t^{*}$ as $n \rightarrow \infty$, then $t^{*}=1$. If not, $0<t^{*}<1$.

Next, we need to prove that $t^{*}=1$. If $0<t^{*}<1$, we should discuss the following two cases.

Case 1: there is an integer $N$ such that $t_{N}=t^{*}$. In this case, we have $t_{n}=t^{*}$ for all $n>N$. Then

$$
\begin{aligned}
u_{n+1} & =T\left(u_{n}, v_{n}\right) \geq T\left(t_{n} v_{n}+\left(t_{n}-1\right) e, t_{n}^{-1} u_{n}+\left(t_{n}^{-1}-1\right) e\right) \\
& =T\left(t^{*} v_{n}+\left(t^{*}-1\right) e,\left(t^{*}\right)^{-1} u_{n}+\left(\left(t^{*}\right)^{-1}-1\right) e\right) \\
& \geq \varphi\left(t^{*}, v_{n}, u_{n}\right) T\left(v_{n}, u_{n}\right)+\left(\varphi\left(t^{*}, v_{n}, u_{n}\right)-1\right) e \\
& \geq \varphi\left(t^{*}, u_{0}, v_{0}\right) T\left(u_{0}, v_{0}\right)+\left(\varphi\left(t^{*}, u_{0}, v_{0}\right)-1\right) e .
\end{aligned}
$$


We can get $t^{*}=t_{n+1} \geq \varphi\left(t^{*}\right)>t^{*}$ from the definition of $t_{n+1}$, which is a contradiction.

Case 2: for all $n, t_{n}<t^{*}$, we have

$$
\begin{aligned}
u_{n+1}= & T\left(u_{n}, v_{n}\right) \geq T\left(t_{n} v_{n}+\left(t_{n}-1\right) e, t_{n}^{-1} u_{n}+\left(t_{n}^{-1}-1\right) e\right) \\
= & T\left(\frac{t_{n}}{t^{*}}\left(t^{*} v_{n}+\left(t^{*}-1\right) e\right)+\left(\frac{t_{n}}{t^{*}}-1\right) e,\left(\frac{t_{n}}{t^{*}}\right)^{-1}\left(\left(t^{*}\right)^{-1} u_{n}+\left(\left(t^{*}\right)^{-1}-1\right) e\right)\right. \\
& \left.+\left(\left(\frac{t_{n}}{t^{*}}\right)^{-1}-1\right) e\right) \\
\geq & \varphi\left(\frac{t_{n}}{t^{*}}, t^{*} v_{n}+\left(t^{*}-1\right) e,\left(t^{*}\right)^{-1} u_{n}+\left(\left(t^{*}\right)^{-1}-1\right) e\right) T\left(t^{*} v_{n}+\left(t^{*}-1\right) e,\left(t^{*}\right)^{-1} u_{n}\right. \\
& \left.+\left(\left(t^{*}\right)^{-1}-1\right) e\right)+\left(\varphi\left(\frac{t_{n}}{t^{*}}, t^{*} v_{n}+\left(t^{*}-1\right) e,\left(t^{*}\right)^{-1} u_{n}+\left(\left(t^{*}\right)^{-1}-1\right) e\right)-1\right) e \\
\geq & \varphi\left(\frac{t_{n}}{t^{*}}, t^{*} v_{n}+\left(t^{*}-1\right) e,\left(t^{*}\right)^{-1} u_{n}+\left(\left(t^{*}\right)^{-1}-1\right) e\right)\left[\varphi\left(t^{*}, v_{n}, u_{n}\right) T\left(v_{n}, u_{n}\right)\right. \\
& \left.+\left(\varphi\left(t^{*}, v_{n}, u_{n}\right)-1\right) e\right] \\
& +\left(\varphi\left(\frac{t_{n}}{t^{*}}, t^{*} v_{n}+\left(t^{*}-1\right) e,\left(t^{*}\right)^{-1} u_{n}+\left(\left(t^{*}\right)^{-1}-1\right) e\right)-1\right) e \\
\geq & \varphi\left(\frac{t_{n}}{t^{*}}, t^{*} u_{0}+\left(t^{*}-1\right) e,\left(t^{*}\right)^{-1} v_{0}+\left(\left(t^{*}\right)^{-1}-1\right) e\right) \varphi\left(t^{*}, u_{0}, v_{0}\right) T\left(v_{n}, u_{n}\right) \\
& +\left(\varphi\left(\frac{t_{n}}{t^{*}}, t^{*} u_{0}+\left(t^{*}-1\right) e,\left(t^{*}\right)^{-1} v_{0}+\left(\left(t^{*}\right)^{-1}-1\right) e\right) \varphi\left(t^{*}, u_{0}, v_{0}\right)-1\right) e .
\end{aligned}
$$

By the definition of $t_{n+1}$, we have

$$
t_{n+1} \geq \varphi\left(\frac{t_{n}}{t^{*}}, t^{*} u_{0}+\left(t^{*}-1\right) e,\left(t^{*}\right)^{-1} v_{0}+\left(\left(t^{*}\right)^{-1}-1\right) e\right) \varphi\left(t^{*}, u_{0}, v_{0}\right) \geq \frac{t_{n}}{t^{*}} \varphi\left(t^{*}, u_{0}, v_{0}\right) .
$$

Let $n \rightarrow \infty$, we have $t^{*} \geq \varphi\left(t^{*}, u_{0}, v_{0}\right)>t^{*}$, which is a contradiction. Consequently $t^{*}=1$. Since $P$ is normal, we have

$$
\left\|u_{n+p}-u_{n}\right\| \leq M\left(1-t_{n}\right)\left\|v_{0}+e\right\|, \quad\left\|v_{n}-v_{n+p}\right\| \leq M\left(1-t_{n}\right)\left\|v_{0}+e\right\|,
$$

where $M$ is the normality constant. Let $n \rightarrow \infty$, we get

$$
\left\|u_{n+p}-u_{n}\right\| \longrightarrow 0, \quad\left\|v_{n}-v_{n+p}\right\| \longrightarrow 0
$$

Therefore $u_{n}$ and $v_{n}$ are Cauchy sequences. Repeating the proof of Lemma 2.3 in Sang and Ren [21], we derive that our conclusions hold.

Lemma 2.7 Let $P$ be a normal cone and $T: P_{h, e} \times P_{h, e} \longrightarrow$ E be a mixed monotone operator. Assume that the condition (i) in Lemma 2.6 is satisfied. In addition, $\varphi(t, u, v)$ is decreasing 
in $u$ and increasing in $v$ for every $t \in(0,1)$. Furthermore, there exists $t_{0} \in(0,1)$ such that

$$
\begin{aligned}
t_{0} h+\left(t_{0}-1\right) e \leq & T(h, h) \\
\leq & \frac{1}{t_{0}} \varphi\left(t_{0}, t_{0}^{-1} h+\left(t_{0}^{-1}-1\right) e, t_{0} h+\left(t_{0}-1\right) e\right) h \\
& +\left[\frac{1}{t_{0}} \varphi\left(t_{0}, t_{0}^{-1} h+\left(t_{0}^{-1}-1\right) e, t_{0} h+\left(t_{0}-1\right) e\right)-1\right] e .
\end{aligned}
$$

Then the conclusions (1), (2), (3) in Lemma 2.6 hold.

Proof As in the proof of Lemma 2.6, we only need to check that $u_{1}=T\left(u_{0}, v_{0}\right) \geq u_{0}$ and $v_{1}=T\left(v_{0}, u_{0}\right) \leq v_{0}$ hold. For every $t \in(0,1)$, since $\varphi(t, u, v)$ is decreasing in $u$ and increasing in $v$, by (2.3), we have

$$
\begin{aligned}
u_{1}= & T\left(u_{0}, v_{0}\right) \\
= & T\left(t_{0}^{k} h+\left(t_{0}^{k}-1\right) e, t_{0}^{-k} h+\left(t_{0}^{-k}-1\right) e\right) \\
\geq & {\left[\varphi ( t _ { 0 } , t _ { 0 } ^ { k - 1 } h + ( t _ { 0 } ^ { k - 1 } - 1 ) e , t _ { 0 } ^ { - k + 1 } h + ( t _ { 0 } ^ { - k + 1 } - 1 ) e ) \varphi \left(t_{0}, t_{0}^{k-2} h+\left(t_{0}^{k-2}-1\right) e, t_{0}^{-k+2} h\right.\right.} \\
& \left.\left.+\left(t_{0}^{-k+2}-1\right) e\right) \cdots \varphi\left(t_{0}, h, h\right)\right] T(h, h)+\left[\varphi \left(t_{0}, t_{0}^{k-1} h+\left(t_{0}^{k-1}-1\right) e, t_{0}^{-k+1} h\right.\right. \\
& \left.\left.+\left(t_{0}^{-k+1}-1\right) e\right) \varphi\left(t_{0}, t_{0}^{k-2} h+\left(t_{0}^{k-2}-1\right) e, t_{0}^{-k+2} h+\left(t_{0}^{-k+2}-1\right) e\right) \cdots \varphi\left(t_{0}, h, h\right)-1\right] e \\
\geq & {\left[\varphi\left(t_{0}, h, h\right)\right]^{k} T(h, h)+\left(\left[\varphi\left(t_{0}, h, h\right)\right]^{k}-1\right) e } \\
\geq & {\left[\varphi\left(t_{0}, h, h\right)\right]^{k}\left[t_{0} h+\left(t_{0}-1\right) e\right]+\left(\left[\varphi\left(t_{0}, h, h\right)\right]^{k}-1\right) e } \\
\geq & t_{0}^{k} h+\left(t_{0}^{k}-1\right) e=x_{k}=u_{0}
\end{aligned}
$$

and

$$
\begin{aligned}
v_{1}= & T\left(v_{0}, u_{0}\right) \\
= & T\left(t_{0}^{-k} h+\left(t_{0}^{-k}-1\right) e, t_{0}^{k} h+\left(t_{0}^{k}-1\right) e\right) \\
\leq & {\left[\varphi ( t _ { 0 } , t _ { 0 } ^ { - k } h + ( t _ { 0 } ^ { - k } - 1 ) e , t _ { 0 } ^ { k } h + ( t _ { 0 } ^ { k } - 1 ) e ) ^ { - 1 } \varphi \left(t_{0}, t_{0}^{-k+1} h+\left(t_{0}^{-k+1}-1\right) e,\right.\right.} \\
& \left.\left.t_{0}^{k-1} h+\left(t_{0}^{k-1}-1\right) e\right)^{-1} \cdots \varphi\left(t_{0}, t_{0}^{-1} h+\left(t_{0}^{-1}-1\right) e, t_{0} h+\left(t_{0}-1\right) e\right)^{-1}\right] T(h, h) \\
& +\left[\varphi ( t _ { 0 } , t _ { 0 } ^ { - k } h + ( t _ { 0 } ^ { - k } - 1 ) e , t _ { 0 } ^ { k } h + ( t _ { 0 } ^ { k } - 1 ) e ) ^ { - 1 } \varphi \left(t_{0}, t_{0}^{-k+1} h+\left(t_{0}^{-k+1}-1\right) e,\right.\right. \\
& \left.\left.t_{0}^{k-1} h+\left(t_{0}^{k-1}-1\right) e\right)^{-1} \cdots \varphi\left(t_{0}, t_{0}^{-1} h+\left(t_{0}^{-1}-1\right) e, t_{0} h+\left(t_{0}-1\right) e\right)^{-1}-1\right] e \\
\leq & t_{0}^{-k+1} \varphi\left(t_{0}, t_{0}^{-1} h+\left(t_{0}^{-1}-1\right) e, t_{0} h+\left(t_{0}-1\right) e\right)^{-1} T(h, h) \\
& +\left[t_{0}^{-k+1} \varphi\left(t_{0}, t_{0}^{-1} h+\left(t_{0}^{-1}-1\right) e, t_{0} h+\left(t_{0}-1\right) e\right)^{-1}-1\right] e \\
\leq & t_{0}^{-k} h+\left(t_{0}^{-k}-1\right) e=y_{k}=v_{0} .
\end{aligned}
$$

The rest of the proof is similar to that of Lemma 2.6, we omit it here.

\section{Main results}

In this section, we will establish the existence and uniqueness of nontrivial solution for the problem (1.1). The main tools are fixed point theorems of an operator equation. 
Theorem 3.1 Let $P$ be a normal cone in $E$, and let $M, N: P_{h, e} \times P_{h, e} \longrightarrow E$ be two mixed monotone operators, and the following conditions are satisfied:

(L1) for all $t \in(0,1)$ and $x, y \in P_{h, e}$, there exists $\psi(t, x, y)>t$ such that

$$
M\left(t x+(t-1) e, t^{-1} y+\left(t^{-1}-1\right) e\right) \geq \psi(t, x, y) M(x, y)+(\psi(t, x, y)-1) e
$$

(L2) for fixed $t \in(0,1)$ and $x \in P_{h, e}, \psi(t, x, y)$ is decreasing in $y$, and for fixed $t \in(0,1)$ and $y \in P_{h, e}, \psi(t, x, y)$ is increasing in $x$;

(L3) for all $t \in(0,1)$ and $x, y \in P_{h, e}$,

$$
N\left(t x+(t-1) e, t^{-1} y+\left(t^{-1}-1\right) e\right) \geq t N(x, y)+(t-1) e ;
$$

(L4) there exists $t_{0} \in(0,1)$ such that

$$
\begin{aligned}
& \frac{t_{0}}{\psi\left(t_{0}, h, h\right)} h+\left(\frac{t_{0}}{\psi\left(t_{0}, h, h\right)}-1\right) e \leq M(h, h) \leq \frac{1}{t_{0}} h+\left(\frac{1}{t_{0}}-1\right) e, \\
& N(h, h) \in P_{h, e}
\end{aligned}
$$

(L5) for all $x, y \in P_{h, e}$, there exists a constant $\delta>0$ such that

$$
M(x, y) \geq \delta N(x, y)+(\delta-1) e .
$$

Then the operator equation $M(x, x)+N(x, x)+e=x$ has a unique solution $x^{*}$ in $P_{h, e}$, and for any initial values $x_{0}, y_{0} \in P_{h, e}$, by setting the sequences $\left\{x_{n}\right\}$ and $\left\{y_{n}\right\}$ as follows:

$$
\begin{array}{ll}
x_{n}=M\left(x_{n-1}, y_{n-1}\right)+N\left(x_{n-1}, y_{n-1}\right)+e, & n=1,2, \ldots, \\
y_{n}=M\left(y_{n-1}, x_{n-1}\right)+N\left(y_{n-1}, x_{n-1}\right)+e, & n=1,2, \ldots,
\end{array}
$$

we have $x_{n} \rightarrow x^{*}$ and $y_{n} \rightarrow x^{*}$ in $E$ as $n \rightarrow \infty$.

Proof For every $x_{i}, y_{i} \in P_{h, e}(i=1,2)$ with $x_{1} \geq x_{2}, y_{1} \leq y_{2}$, the mixed monotone properties of $M(x, y)$ and $N(x, y)$ lead to

$$
M\left(x_{1}, y_{1}\right) \geq M\left(x_{2}, y_{2}\right), \quad N\left(x_{1}, y_{1}\right) \geq N\left(x_{2}, y_{2}\right) .
$$

Now we define the operator $T: P_{h, e} \times P_{h, e} \rightarrow E$ by

$$
T(x, y)=M(x, y)+N(x, y)+e, \quad \text { for all } x, y \in P_{h, e} .
$$

We have

$$
T\left(x_{1}, y_{1}\right)=M\left(x_{1}, y_{1}\right)+N\left(x_{1}, y_{1}\right)+e \geq M\left(x_{2}, y_{2}\right)+N\left(x_{2}, y_{2}\right)+e=T\left(x_{2}, y_{2}\right) .
$$

Thus, $T$ is a mixed monotone operator. Note that $N(h, h) \in P_{h, e}$, there exist $a_{1}, a_{2} \in P_{h, e}$ such that

$$
a_{1} h+\left(a_{1}-1\right) e \leq N(h, h) \leq a_{2} h+\left(a_{2}-1\right) e .
$$


From (3.1), we have

$$
T(h, h)=M(h, h)+N(h, h)+e .
$$

By the condition (L4), we obtain

$$
\begin{aligned}
T(h, h) & \geq \frac{t_{0}}{\psi\left(t_{0}, h, h\right)} h+\left(\frac{t_{0}}{\psi\left(t_{0}, h, h\right)}-1\right) e+a_{1} h+\left(a_{1}-1\right) e \\
& =\left(\frac{t_{0}}{\psi\left(t_{0}, h, h\right)}+a_{1}\right) h+\left(\frac{t_{0}}{\psi\left(t_{0}, h, h\right)}+a_{1}-1\right) e
\end{aligned}
$$

and

$$
\begin{aligned}
T(h, h) & \leq \frac{1}{t_{0}} h+\left(\frac{1}{t_{0}}-1\right) e+a_{2} h+\left(a_{2}-1\right) e \\
& =\left(\frac{1}{t_{0}}+a_{2}\right) h+\left(\frac{1}{t_{0}}+a_{2}-1\right) e .
\end{aligned}
$$

Hence, the condition (iii) in Lemma 2.6 is proved. Next, by the condition (L4), we have

$$
\begin{aligned}
& M(x, y)+\delta M(x, y) \geq \delta N(x, y)+(\delta-1) e+\delta M(x, y) \\
& M(x, y) \geq \frac{\delta}{1+\delta} T(x, y)-\frac{e}{1+\delta} .
\end{aligned}
$$

By conditions (L1), (L3), (3.1) and (3.2), for every $x, y \in P_{h, e}$, we obtain

$$
\begin{aligned}
T( & \left.t x+(t-1) e, t^{-1} y+\left(t^{-1}-1\right) e\right)-t T(x, y) \\
= & M\left(t x+(t-1) e, t^{-1} y+\left(t^{-1}-1\right) e\right)+N\left(t x+(t-1) e, t^{-1} y+\left(t^{-1}-1\right) e\right)+e \\
& -t(M(x, y)+N(x, y)+e) \\
\geq & \psi(t, x, y) M(x, y)+(\psi(t, x, y)-1) e+t N(x, y)+(t-1) e+e-t M(x, y) \\
& -t N(x, y)-t e \\
= & (\psi(t, x, y)-t) M(x, y)+(\psi(t, x, y)-1) e \\
\geq & (\psi(t, x, y)-t)\left(\frac{\delta}{1+\delta} T(x, y)-\frac{e}{1+\delta}\right)+(\psi(t, x, y)-1) e \\
= & \frac{\delta(\psi(t, x, y)-t)}{1+\delta} T(x, y)+\left(\psi(t, x, y)-1-\frac{\psi(t, x, y)-t}{1+\delta}\right) e .
\end{aligned}
$$

Therefore

$$
\begin{aligned}
& T\left(t x+(t-1) e, t^{-1} y+\left(t^{-1}-1\right) e\right) \\
& \quad \geq\left(\frac{\delta(\psi(t, x, y)-t)}{1+\delta}+t\right) T(x, y)+\left(\psi(t, x, y)-1-\frac{\psi(t, x, y)-t}{1+\delta}\right) e \\
& \quad=\frac{\delta \psi(t, x, y)+t}{1+\delta} T(x, y)+\left(\frac{\delta \psi(t, x, y)+t}{1+\delta}-1\right) e .
\end{aligned}
$$


Let $\varphi(t, x, y)=\frac{\delta \psi(t, x, y)+t}{1+\delta}$. Then $\varphi(t, x, y)>t, t \in(0,1)$, together with (3.3), we obtain

$$
T\left(t x+(t-1) e, t^{-1} y+\left(t^{-1}-1\right) e\right) \geq \varphi(t, x, y) T(x, y)+(\varphi(t, x, y)-1) e, \quad \forall x, y \in P_{h, e}
$$

Thus condition (i) in Lemma 2.6 is proved. By (L2), for every $x_{1} \geq x_{2}$ and $y_{1} \leq y_{2}$ with $x_{i}, y_{i} \in P_{h, e}, i=1,2$, we have

$$
\psi\left(t, x_{1}, y_{1}\right) \geq \psi\left(t, x_{2}, y_{2}\right)
$$

Therefore

$$
\varphi\left(t, x_{1}, y_{1}\right)=\frac{\delta \psi\left(t, x_{1}, y_{1}\right)+t}{1+\delta} \geq \frac{\delta \psi\left(t, x_{2}, y_{2}\right)+t}{1+\delta}=\varphi\left(t, x_{2}, y_{2}\right)
$$

Thus we deduce condition (ii) in Lemma 2.6 to be met. According to Lemma 2.6, we get the conclusions of Theorem 3.1.

In terms of Lemma 2.7, we can establish the following theorem, which is parallel with Theorem 3.1.

Theorem 3.1' Let $P$ be a normal cone in $E$, and $M, N: P_{h, e} \times P_{h, e} \rightarrow E$ be two mixed monotone operators. The assumptions (L1), (L3) and (L5) in Theorem 3.1 are satisfied. Furthermore, for fixed $t \in(0,1)$ and $x \in P_{h, e}, \psi(t, x, y)$ is increasing in $y$, and for fixed $t \in$ $(0,1)$ and $y \in P_{h, e}, \psi(t, x, y)$ is decreasing in $x$. In addition, $N(h, h) \in P_{h, e}$, and there exists $t_{0} \in(0,1)$ such that

$$
\begin{aligned}
t_{0} h+\left(t_{0}-1\right) e \leq & M(h, h) \\
\leq & \frac{1}{t_{0}} \psi\left(t_{0}, t_{0}^{-1} h+\left(t_{0}^{-1}-1\right) e, t_{0} h+\left(t_{0}-1\right) e\right) h \\
& +\left[\frac{1}{t_{0}} \psi\left(t_{0}, t_{0}^{-1} h+\left(t_{0}^{-1}-1\right) e, t_{0} h+\left(t_{0}-1\right) e\right)-1\right] e .
\end{aligned}
$$

Then the conclusions of Theorem 3.1 hold.

Define $E=\left\{x \mid x \in C[0,1], D_{0^{+}}^{v} x \in C[0,1]\right\}$. Then $E$ is a Banach space with an order relation $u \leq v$ if $u(t) \leq v(t), D_{0^{+}}^{v} u(t) \leq D_{0^{+}}^{v} v(t)$. Let $P \subset E$ be defined by $P=\{x \in E \mid x(t) \geq$ $\left.0, D_{0^{+}}^{v} x(t) \geq 0\right\}$ for all $t \in[0,1]$. It is clear that $P$ is a normal cone. Let

$$
e(t)=b \int_{0}^{1} G(t, s)\left(\frac{s^{\beta-1}-s^{\beta}}{\Gamma(\beta+1)}+\frac{\sum_{i=1}^{m-2} \zeta_{i}\left(\eta_{i}^{\beta-1}-\eta_{i}^{\beta}\right) s^{\beta-1}}{B \Gamma(\beta+1)}\right) d s, \quad t \in[0,1] .
$$

\section{Theorem 3.2 Assume that}

(H1) $f, g:[0,1] \times\left[-e^{*},+\infty\right) \times\left[-e^{*},+\infty\right) \rightarrow(-\infty,+\infty)$ are continuous. For every $t \in[0,1], g(t, 0, \mathcal{K}(L)) \geq 0$ with $g(t, 0, \mathcal{K}(L)) \not \equiv 0$ where $L \geq \frac{b D F}{\beta \Gamma(\alpha) \Gamma(\beta)}$ and $e^{*}=\max \{e(t): t \in[0,1]\} ;$

(H2) for fixed $t \in[0,1]$ and $y \in\left[-e^{*},+\infty\right), f(t, x, y), g(t, x, y)$ are increasing in $x \in\left[-e^{*},+\infty\right)$; for fixed $t \in[0,1]$ and $x \in\left[-e^{*},+\infty\right), f(t, x, y), g(t, x, y)$ are decreasing in $y \in\left[-e^{*},+\infty\right)$; 
(H3) for all $\lambda \in(0,1)$, there exists $\psi(\lambda, x, y) \in(\lambda, 1)$ such that for all $t \in[0,1]$,

(a) $f\left(t, \lambda x+(\lambda-1) \rho_{1}, \lambda^{-1} D_{0^{+}}^{v} y+\left(\lambda^{-1}-1\right) \rho_{2}\right) \geq \psi(\lambda, x, y) f\left(t, x, D_{0^{+}}^{v} y\right)$,

(b) $g\left(t, \lambda x+(\lambda-1) \rho_{3}, \lambda^{-1} y+\left(\lambda^{-1}-1\right) \rho_{3}\right) \geq \lambda g(t, x, y)$, where $x, y \in\left[-e^{*},+\infty\right)$, $\rho_{1}, \rho_{3} \in\left[0, e^{*}\right]$, and $\rho_{2} \in\left[0, e_{*}\right]$ with $e_{*}=\max \left\{D_{0^{+}}^{v} e(t): t \in[0,1]\right\}$,

(c) for fixed $t \in[0,1]$ and $y \in P_{h, e}, \psi(\lambda, x, y)$ are increasing in $x \in P_{h, e}$ and for fixed $t \in[0,1]$ and $x \in P_{h, e}, \psi(\lambda, x, y)$ are decreasing in $y \in P_{h, e}$;

(H4) for all $t \in[0,1], x, y \in\left[-e^{*},+\infty\right)$, there exists $\delta>0$ such that

$$
f(t, x, y) \geq \delta g(t, x, 0)
$$

(H5) $\mathcal{K}: C[0,1] \rightarrow C[0,1]$ and satisfies

(a) $\mathcal{K} u \geq 0$ for every $u \in P_{h, e}$,

(b) for $u, v \in P_{h, e}, u \leq v \Longrightarrow \mathcal{K} u \leq \mathcal{K} v$,

(c) for all $\lambda \in(0,1)$ and $u \in P_{h, e}$,

$$
\mathcal{K}(\lambda u+(\lambda-1) \hat{e}) \geq \lambda \mathcal{K}(u)+(\lambda-1) \hat{e}, \quad \hat{e} \in\left[0, e^{*}\right]
$$

(H6) there exists $t_{0} \in(0,1)$ such that

$$
\begin{aligned}
& \frac{t_{0}}{\psi\left(t_{0}, h, h\right)} h(t)+\frac{t_{0}}{\psi\left(t_{0}, h, h\right)} e(t) \\
& \quad \leq \int_{0}^{1} G(t, s)\left(\int_{0}^{1} H(s, \tau) f\left(\tau, h(\tau), D_{0^{+}}^{v} h(\tau)\right) d \tau\right) d s \\
& \quad \leq \frac{1}{t_{0}} h(t)+\frac{1}{t_{0}} e(t) .
\end{aligned}
$$

Then the problem (1.1) has a unique solution $u^{*}$ in $P_{h, e}$, where $h(t)=L t^{\alpha-1}$, for all $t \in[0,1]$. We can construct the following sequences:

$$
\begin{aligned}
\omega_{n}(t)= & \int_{0}^{1} G(t, s)\left(\int_{0}^{1} H(s, \tau) f\left(\tau, \omega_{n-1}(\tau), D_{0^{+}}^{v} \sigma_{n-1}(\tau)\right) d \tau\right) d s \\
& +\int_{0}^{1} G(t, s)\left(\int_{0}^{1} H(s, \tau) g\left(\tau, \omega_{n-1}(\tau),\left(\mathcal{K} \sigma_{n-1}\right)(\tau)\right) d \tau\right) d s-e(t), \quad n=1,2, \ldots \\
\sigma_{n}(t)= & \int_{0}^{1} G(t, s)\left(\int_{0}^{1} H(s, \tau) f\left(\tau, \sigma_{n-1}(\tau), D_{0^{+}}^{v} \omega_{n-1}(\tau)\right) d \tau\right) d s \\
& +\int_{0}^{1} G(t, s)\left(\int_{0}^{1} H(s, \tau) g\left(\tau, \sigma_{n-1},\left(\mathcal{K} \omega_{n-1}\right)(\tau)\right) d \tau\right) d s-e(t), \quad n=1,2, \ldots
\end{aligned}
$$

for every initial value $\omega_{0}, \sigma_{0} \in P_{h, e}$,we have $\omega_{n} \rightarrow u^{*}$ and $\sigma_{n} \rightarrow u^{*}$ as $n \rightarrow \infty$.

Proof By [3], we have

$$
\begin{aligned}
\int_{0}^{1} H(s, \tau) d \tau & =\int_{0}^{1} H_{1}(s, \tau) d \tau+\int_{0}^{1} H_{2}(s, \tau) d \tau \\
& =\frac{s^{\beta-1}-s^{\beta}}{\Gamma(\beta+1)}+\frac{\sum_{i=1}^{m-2} \zeta_{i}\left(\eta_{i}^{\beta-1}-\eta_{i}^{\beta}\right) s^{\beta-1}}{B \Gamma(\beta+1)} .
\end{aligned}
$$


Furthermore, it follows from Lemmas 2.4 and 2.5 that

$$
\begin{aligned}
0 & <e(t) \leq b \int_{0}^{1} \frac{D t^{\alpha-1}}{\Gamma(\alpha)}\left(\int_{0}^{1} \frac{F s^{\beta-1}}{\Gamma(\beta)} d \tau\right) d s \\
& =\frac{b D F}{\Gamma(\alpha) \Gamma(\beta)} t^{\alpha-1} \int_{0}^{1} s^{\beta-1} d s \\
& =\frac{b D F}{\beta \Gamma(\alpha) \Gamma(\beta)} t^{\alpha-1} \\
& \leq L t^{\alpha-1}=h(t)
\end{aligned}
$$

where $L \geq \frac{b D F}{\beta \Gamma(\alpha) \Gamma(\beta)}$. Hence, $0<e(t) \leq h(t)$. By Lemma 2.3, we find that the problem (1.1) has the following expression:

$$
\begin{aligned}
u(t)= & \int_{0}^{1} G(t, s)\left(\int_{0}^{1} H(s, \tau)\left(f\left(\tau, u(\tau), D_{0^{+}}^{v} u(\tau)\right)+g(\tau, u(\tau),(\mathcal{K} u)(\tau))-b\right) d \tau\right) d s \\
= & \int_{0}^{1} G(t, s) \int_{0}^{1} H(s, \tau) f\left(\tau, u(\tau), D_{0^{+}}^{v} u(\tau)\right) d \tau d s \\
& +\int_{0}^{1} G(t, s) \int_{0}^{1} H(s, \tau) g(\tau, u(\tau),(\mathcal{K} u)(\tau)) d \tau d s \\
& -\int_{0}^{1} G(t, s) b \int_{0}^{1} H(s, \tau) d \tau d s \\
= & \int_{0}^{1} G(t, s) \int_{0}^{1} H(s, \tau) f\left(\tau, u(\tau), D_{0^{+}}^{v} u(\tau)\right) d \tau d s-e(t) \\
& +\int_{0}^{1} G(t, s) \int_{0}^{1} H(s, \tau) g(\tau, u(\tau),(\mathcal{K} u)(\tau)) d \tau d s-e(t)+e(t) .
\end{aligned}
$$

For every $t \in[0,1]$ and $u, v \in P_{h, e}$, we consider the following operators:

$$
M(u, v)(t)=\int_{0}^{1} G(t, s) \int_{0}^{1} H(s, \tau) f\left(\tau, u(\tau), D_{0^{+}}^{v} v(\tau)\right) d \tau d s-e(t)
$$

and

$$
N(u, v)(t)=\int_{0}^{1} G(t, s) \int_{0}^{1} H(s, \tau) g(\tau, u(\tau),(\mathcal{K} v)(\tau)) d \tau d s-e(t)
$$

Clearly, $u(t)$ is the solution of problem (1.1) is equivalent to $u$ is the fixed point of $M(u, v)(t)+N(u, v)(t)+e$. By (3.4) and (3.5), we get

$$
\begin{aligned}
& D_{0^{+}}^{v} M(u, v)(t)=\int_{0}^{1} D_{0^{+}}^{v} G(t, s) \int_{0}^{1} H(s, \tau) f\left(\tau, u(\tau), D_{0^{+}}^{v} v(\tau)\right) d \tau d s-D_{0^{+}}^{v} e(t), \\
& D_{0^{+}}^{v} N(u, v)(t)=\int_{0}^{1} D_{0^{+}}^{v} G(t, s) \int_{0}^{1} H(s, \tau) g(\tau, u(\tau),(\mathcal{K} v)(\tau)) d \tau d s-D_{0^{+}}^{v} e(t) .
\end{aligned}
$$


(1) Firstly, we show that $M, N: P_{h . e} \times P_{\text {h.e }} \rightarrow E$ are two mixed monotone operators. By (H1) and (H2), for every $u_{i}, v_{i} \in P_{h, e}(i=1,2)$ with $u_{1} \geq u_{2}, v_{1} \leq v_{2}$, we have

$$
\begin{aligned}
M\left(u_{1}, v_{1}\right)(t) & =\int_{0}^{1} G(t, s) \int_{0}^{1} H(s, \tau) f\left(\tau, u_{1}(\tau), D_{0^{+}}^{v} \nu_{1}(\tau)\right) d \tau d s-e(t) \\
& \geq \int_{0}^{1} G(t, s) \int_{0}^{1} H(s, \tau) f\left(\tau, u_{2}(\tau), D_{0^{+}}^{v} \nu_{2}(\tau)\right) d \tau d s-e(t)=M\left(u_{2}, v_{2}\right)(t)
\end{aligned}
$$

and

$$
\begin{aligned}
D_{0^{+}}^{v} M\left(u_{1}, v_{1}\right)(t) & =\int_{0}^{1} D_{0^{+}}^{v} G(t, s) \int_{0}^{1} H(s, \tau) f\left(\tau, u_{1}(\tau), D_{0^{+}}^{v} v_{1}(\tau)\right) d \tau d s-D_{0^{+}}^{v} e(t) \\
& \geq \int_{0}^{1} D_{0^{+}}^{v} G(t, s) \int_{0}^{1} H(s, \tau) f\left(\tau, u_{2}(\tau), D_{0^{+}}^{v} v_{2}(\tau)\right) d \tau d s-D_{0^{+}}^{v} e(t) \\
& =D_{0^{+}}^{v} M\left(u_{2}, v_{2}\right)(t) .
\end{aligned}
$$

Hence, $M$ is a mixed monotone operator. Similarly, we deduce

$$
\begin{aligned}
N\left(u_{1}, v_{1}\right)(t) & =\int_{0}^{1} G(t, s) \int_{0}^{1} H(s, \tau) g\left(\tau, u_{1}(\tau),\left(\mathcal{K} v_{1}\right)(\tau)\right) d \tau d s-e(t) \\
& \geq \int_{0}^{1} G(t, s) \int_{0}^{1} H(s, \tau) g\left(\tau, u_{2}(\tau),\left(\mathcal{K} v_{2}\right)(\tau)\right) d \tau d s-e(t)=N\left(u_{2}, v_{2}\right)(t)
\end{aligned}
$$

and

$$
\begin{aligned}
D_{0^{+}}^{v} N\left(u_{1}, v_{1}\right)(t) & =\int_{0}^{1} D_{0^{+}}^{v} G(t, s) \int_{0}^{1} H(s, \tau) g\left(\tau, u_{1}(\tau),\left(\mathcal{K} v_{1}\right)(\tau)\right) d \tau d s-D_{0^{+}}^{v} e(t) \\
& \geq \int_{0}^{1} D_{0^{+}}^{v} G(t, s) \int_{0}^{1} H(s, \tau) g\left(\tau, u_{2}(\tau),\left(\mathcal{K} v_{2}\right)(\tau)\right) d \tau d s-D_{0^{+}}^{v} e(t) \\
& =D_{0^{+}}^{v} N\left(u_{2}, v_{2}\right)(t) .
\end{aligned}
$$

Thus, $N$ is also a mixed monotone operator.

(2) Next, by (H3), for every $t \in[0,1]$ and $\lambda \in(0,1)$, there exists $\psi(\lambda, u, v) \in(\lambda, 1)$ such that, for every $u, v \in P_{h, e}$, we get

$$
\begin{aligned}
M( & \left.\lambda u+(\lambda-1) e, \lambda^{-1} v+\left(\lambda^{-1}-1\right) e\right)(t) \\
= & \int_{0}^{1} G(t, s) \int_{0}^{1} H(s, \tau) f\left(\tau, \lambda u+(\lambda-1) e, D_{0^{+}}^{v}\left(\lambda^{-1} v+\left(\lambda^{-1}-1\right) e\right)\right) d \tau d s-e(t) \\
= & \int_{0}^{1} G(t, s) \int_{0}^{1} H(s, \tau) f\left(\tau, \lambda u+(\lambda-1) e, \lambda^{-1} D_{0^{+}}^{v} v+\left(\lambda^{-1}-1\right) D_{0^{+}}^{v} e\right) d \tau d s-e(t) \\
\geq & \int_{0}^{1} G(t, s) \int_{0}^{1} H(s, \tau) \psi(\lambda, u(\tau), v(\tau)) f\left(\tau, u(\tau), D_{0^{+}}^{v} v(\tau)\right) d \tau d s-e(t) \\
& +\psi(\lambda, u, v) e(t)-\psi(\lambda, u, v) e(t) \\
= & \psi(\lambda, u, v) M(u, v)(t)+(\psi(\lambda, u, v)-1) e(t)
\end{aligned}
$$


and

$$
\begin{array}{rl}
D_{0^{+}}^{v} & M\left(\lambda u+(\lambda-1) e, \lambda^{-1} v+\left(\lambda^{-1}-1\right) e\right)(t) \\
= & \int_{0}^{1} D_{0^{+}}^{v} G(t, s) \int_{0}^{1} H(s, \tau) f\left(\tau, \lambda u+(\lambda-1) e, D_{0^{+}}^{v}\left(\lambda^{-1} v+\left(\lambda^{-1}-1\right) e\right)\right) d \tau d s \\
& -D_{0^{+}}^{v} e(t) \\
= & \int_{0}^{1} D_{0^{+}}^{v} G(t, s) \int_{0}^{1} H(s, \tau) f\left(\tau, \lambda u+(\lambda-1) e, \lambda^{-1} D_{0^{+}}^{v} v+\left(\lambda^{-1}-1\right) D_{0^{+}}^{v} e\right) d \tau d s \\
& -D_{0^{+}}^{v} e(t) \\
\geq & \int_{0}^{1} D_{0^{+}}^{v} G(t, s) \int_{0}^{1} H(s, \tau) \psi(\lambda, u(\tau), v(\tau)) f\left(\tau, u(\tau), D_{0^{+}}^{v} v(\tau)\right) d \tau d s-D_{0^{+}}^{v} e(t) \\
& +\psi(\lambda, u, v) D_{0^{+}}^{v} e(t)-\psi(\lambda, u, v) D_{0^{+}}^{v} e(t) \\
= & \psi(\lambda, u, v) D_{0^{+}}^{v} M(u, v)(t)+(\psi(\lambda, u, v)-1) D_{0^{+}}^{v} e(t) .
\end{array}
$$

Thus, $M\left(\lambda u+(\lambda-1) e, \lambda^{-1} v+\left(\lambda^{-1}-1\right) e\right) \geq \psi(\lambda, u, v) M(u, v)+(\psi(\lambda, u, v)-1) e$.

In view of $(\mathrm{H} 3)(\mathrm{b})$ and $(\mathrm{H} 5)$, we derive

$$
\begin{aligned}
& \mathcal{K}\left(\lambda^{-1} u+\left(\lambda^{-1}-1\right) e\right) \leq \lambda^{-1}(\mathcal{K} u)+\left(\lambda^{-1}-1\right) e \\
& N\left(\lambda u+(\lambda-1) e, \lambda^{-1} v+\left(\lambda^{-1}-1\right) e\right)(t) \\
& \quad=\int_{0}^{1} G(t, s) \int_{0}^{1} H(s, \tau) g\left(\tau, \lambda u+(\lambda-1) e,\left(\mathcal{K}\left(\lambda^{-1} v+\left(\lambda^{-1}-1\right) e\right)\right) d \tau d s-e(t)\right. \\
& \quad \geq \int_{0}^{1} G(t, s) \int_{0}^{1} H(s, \tau) g\left(\tau, \lambda u+(\lambda-1) e, \lambda^{-1}(\mathcal{K} v)+\left(\lambda^{-1}-1\right) e\right) d \tau d s-e(t) \\
& \quad \geq \int_{0}^{1} G(t, s) \int_{0}^{1} H(s, \tau) \lambda g(\tau, u(\tau),(\mathcal{K} v)(\tau)) d \tau d s-e(t) \\
& \quad=\lambda \int_{0}^{1} G(t, s) \int_{0}^{1} H(s, \tau) g(\tau, u(\tau),(\mathcal{K} v)(\tau)) d \tau d s-e(t)+\lambda e(t)-\lambda e(t) \\
& \quad=\lambda N(u, v)(t)+(\lambda-1) e(t),
\end{aligned}
$$

and

$$
\begin{array}{rl}
D_{0^{+}}^{v} & N\left(\lambda u+(\lambda-1) e, \lambda^{-1} v+\left(\lambda^{-1}-1\right) e\right)(t) \\
& =\int_{0}^{1} D_{0^{+}}^{v} G(t, s) \int_{0}^{1} H(s, \tau) g\left(\tau, \lambda u+(\lambda-1) e,\left(\mathcal{K}\left(\lambda^{-1} v+\left(\lambda^{-1}-1\right) e\right)\right) d \tau d s-D_{0^{+}}^{v} e(t)\right. \\
& \geq \int_{0}^{1} D_{0^{+}}^{v} G(t, s) \int_{0}^{1} H(s, \tau) g\left(\tau, \lambda u+(\lambda-1) e, \lambda^{-1}(\mathcal{K} v)+\left(\lambda^{-1}-1\right) e\right) d \tau d s-D_{0^{+}}^{v} e(t) \\
& \geq \int_{0}^{1} D_{0^{+}}^{v} G(t, s) \int_{0}^{1} H(s, \tau) \lambda g(\tau, u(\tau),(\mathcal{K} v)(\tau)) d \tau d s-D_{0^{+}}^{v} e(t) \\
& =\lambda \int_{0}^{1} D_{0^{+}}^{v} G(t, s) \int_{0}^{1} H(s, \tau) g(\tau, u(\tau),(\mathcal{K} v)(\tau)) d \tau d s
\end{array}
$$




$$
\begin{aligned}
& -D_{0^{+}}^{v} e(t)+\lambda D_{0^{+}}^{v} e(t)-\lambda D_{0^{+}}^{v} e(t) \\
= & \lambda D_{0^{+}}^{v} N(u, v)(t)+(\lambda-1) D_{0^{+}}^{v} e(t) .
\end{aligned}
$$

Thus, $N\left(\lambda u+(\lambda-1) e, \lambda^{-1} v+\left(\lambda^{-1}-1\right) e\right) \geq \lambda N(u, v)+(\lambda-1) e$.

(3) In view of (H6), we have

$$
\begin{aligned}
M(h, h)(t) & =\int_{0}^{1} G(t, s) \int_{0}^{1} H(s, \tau) f\left(\tau, h(\tau), D_{0^{+}}^{v} h(\tau)\right) d \tau d s-e(t) \\
& \leq \frac{1}{t_{0}} h(t)+\left(\frac{1}{t_{0}}-1\right) e(t), \\
M(h, h)(t) & \geq \frac{t_{0}}{\psi\left(t_{0}, h, h\right)} h(t)+\left(\frac{t_{0}}{\psi\left(t_{0}, h, h\right)}-1\right) e(t),
\end{aligned}
$$

and

$$
\begin{aligned}
D_{0^{+}}^{v} M(h, h)(t) & =\int_{0}^{1} D_{0^{+}}^{v} G(t, s) \int_{0}^{1} H(s, \tau) f\left(\tau, h(\tau), D_{0^{+}}^{v} h(\tau)\right) d \tau d s-D_{0^{+}}^{v} e(t) \\
& \leq D_{0^{+}}^{v} \frac{1}{t_{0}} h(t)+\left(\frac{1}{t_{0}}-1\right) D_{0^{+}}^{v} e(t), \\
D_{0^{+}}^{v} M(h, h)(t) & \geq D_{0^{+}}^{v} \frac{t_{0}}{\psi\left(t_{0}, h, h\right)} h(t)+\left(\frac{t_{0}}{\psi\left(t_{0}, h, h\right)}-1\right) D_{0^{+}}^{v} e(t) .
\end{aligned}
$$

Thus,

$$
\frac{t_{0}}{\psi\left(t_{0}, h, h\right)} h+\left(\frac{t_{0}}{\psi\left(t_{0}, h, h\right)}-1\right) e \leq M(h, h) \leq \frac{\psi\left(t_{0}, h, h\right)}{t_{0}} h+\left(\frac{\psi\left(t_{0}, h, h\right)}{t_{0}}-1\right) e .
$$

Next we show that $N(h, h) \in P_{h, e}$. It suffices to prove that $N(h, h)+e \in P_{h}$. From Lemma 2.4 and the condition (H2), we have

$$
\begin{aligned}
N(h, h)(t)+e(t) & =\int_{0}^{1} G(t, s) \int_{0}^{1} H(s, \tau) g(\tau, h(\tau),(\mathcal{K} h)(\tau)) d \tau d s \\
& \leq \int_{0}^{1} \frac{D t^{\alpha-1}}{\Gamma(\alpha)} \int_{0}^{1} H(s, \tau) g\left(\tau, L \tau^{\alpha-1}, \mathcal{K}\left(L \tau^{\alpha-1}\right)\right) d \tau d s \\
& \leq \frac{D t^{\alpha-1}}{\Gamma(\alpha)} \int_{0}^{1} \int_{0}^{1} H(s, \tau) g(\tau, L, 0) d \tau d s \\
& =\frac{D h(t)}{L \Gamma(\alpha)} \int_{0}^{1} \int_{0}^{1} H(s, \tau) g(\tau, L, 0) d \tau d s, \\
N(h, h)(t)+e(t) & =\int_{0}^{1} G(t, s) \int_{0}^{1} H(s, \tau) g(\tau, h(\tau),(\mathcal{K} h)(\tau)) d \tau d s \\
& \geq \int_{0}^{1} \frac{C(s)}{\Gamma(\alpha)} t^{\alpha-1} \int_{0}^{1} H(s, \tau) g\left(\tau, L \tau^{\alpha-1}, \mathcal{K}\left(L \tau^{\alpha-1}\right)\right) d \tau d s \\
& \geq \frac{t^{\alpha-1}}{\Gamma(\alpha)} \int_{0}^{1} C(s) \int_{0}^{1} H(s, \tau) g(\tau, 0, \mathcal{K}(L)) d \tau d s \\
& =\frac{h(t)}{L \Gamma(\alpha)} \int_{0}^{1} C(s) \int_{0}^{1} H(s, \tau) g(\tau, 0, \mathcal{K}(L)) d \tau d s,
\end{aligned}
$$


and

$$
\begin{aligned}
D_{0^{+}}^{v} N(h, h)(t)+D_{0^{+}}^{v} e(t) & =\int_{0}^{1} D_{0^{+}}^{v} G(t, s) \int_{0}^{1} H(s, \tau) g(\tau, h(\tau),(\mathcal{K} h)(\tau)) d \tau d s \\
& \leq \int_{0}^{1} \frac{D t^{\alpha-\nu-1}}{\Gamma(\alpha-v)} \int_{0}^{1} H(s, \tau) g\left(\tau, L \tau^{\alpha-1}, \mathcal{K}\left(L \tau^{\alpha-1}\right)\right) d \tau d s \\
& \leq \frac{D t^{\alpha-\nu-1}}{\Gamma(\alpha-v)} \int_{0}^{1} \int_{0}^{1} H(s, \tau) g(\tau, L, 0) d \tau d s \\
& =\frac{D D_{0^{+}}^{v} h(t)}{L \Gamma(\alpha)} \int_{0}^{1} \int_{0}^{1} H(s, \tau) g(\tau, L, 0) d \tau d s, \\
D_{0^{+}}^{v} N(h, h)(t)+D_{0^{+}}^{v} e(t) & =\int_{0}^{1} D_{0^{+}}^{v} G(t, s) \int_{0}^{1} H(s, \tau) g(\tau, h(\tau),(\mathcal{K} h)(\tau)) d \tau d s \\
& \geq \int_{0}^{1} \frac{C(s)}{\Gamma(\alpha-v)} t^{\alpha-\nu-1} \int_{0}^{1} H(s, \tau) g\left(\tau, L \tau^{\alpha-1}, \mathcal{K}\left(L \tau^{\alpha-1}\right)\right) d \tau d s \\
& \geq \frac{t^{\alpha-\nu-1}}{\Gamma(\alpha-v)} \int_{0}^{1} C(s) \int_{0}^{1} H(s, \tau) g(\tau, 0, \mathcal{K}(L)) d \tau d s \\
& =\frac{D_{0^{+}}^{v} h(t)}{L \Gamma(\alpha)} \int_{0}^{1} C(s) \int_{0}^{1} H(s, \tau) g(\tau, 0, \mathcal{K}(L)) d \tau d s .
\end{aligned}
$$

Let

$$
\begin{aligned}
& l_{1}=\frac{D}{L \Gamma(\alpha)} \int_{0}^{1} \int_{0}^{1} H(s, \tau) g(\tau, L, 0) d \tau d s \\
& l_{2}=\frac{1}{L \Gamma(\alpha)} \int_{0}^{1} C(s) \int_{0}^{1} H(s, \tau) g(\tau, 0, \mathcal{K}(L)) d \tau d s .
\end{aligned}
$$

Then $l_{2} h \leq N(h, h)+e \leq l_{1} h$, thus $N(h, h) \in P_{h, e}$. Therefore, the condition (L4) of Theorem 3.1 is proved.

(4) For every $u, v \in P_{h, e}$ and $t \in[0,1]$, we derive that

$$
\begin{aligned}
M(u, v)(t) & =\int_{0}^{1} G(t, s) \int_{0}^{1} H(s, \tau) f\left(\tau, u(\tau), D_{0^{+}}^{v} v(\tau)\right) d \tau d s-e(t) \\
& \geq \int_{0}^{1} G(t, s) \int_{0}^{1} H(s, \tau) \delta g(\tau, u(\tau), 0) d \tau d s-e(t) \\
& \geq \int_{0}^{1} G(t, s) \int_{0}^{1} H(s, \tau) \delta g(\tau, u(\tau),(\mathcal{K} v)(\tau)) d \tau d s-e(t) \\
& =\delta \int_{0}^{1} G(t, s) \int_{0}^{1} H(s, \tau) g(\tau, u(\tau),(\mathcal{K} v)(\tau)) d \tau d s-e(t)+\delta e(t)-\delta e(t) \\
& =\delta N(u, v)(t)+(\delta-1) e(t)
\end{aligned}
$$

and

$$
\begin{aligned}
D_{0^{+}}^{v} M(u, v)(t) & =\int_{0}^{1} D_{0^{+}}^{v} G(t, s) \int_{0}^{1} H(s, \tau) f\left(\tau, u(\tau), D_{0^{+}}^{v} \nu(\tau)\right) d \tau d s-D_{0^{+}}^{v} e(t) \\
& \geq \int_{0}^{1} D_{0^{+}}^{v} G(t, s) \int_{0}^{1} H(s, \tau) \delta g(\tau, u(\tau), 0) d \tau d s-D_{0^{+}}^{v} e(t)
\end{aligned}
$$




$$
\begin{aligned}
& \geq \int_{0}^{1} D_{0^{+}}^{v} G(t, s) \int_{0}^{1} H(s, \tau) \delta g(\tau, u(\tau),(\mathcal{K} v)(\tau)) d \tau d s-D_{0^{+}}^{v} e(t) \\
& =\delta \int_{0}^{1} G(t, s) \int_{0}^{1} H(s, \tau) g(\tau, u(\tau),(\mathcal{K} v)(\tau)) d \tau d s-D_{0^{+}}^{v} e(t) \\
& +\delta D_{0^{+}}^{v} e(t)-\delta D_{0^{+}}^{v} e(t) \\
& =\delta D_{0^{+}}^{v} N(u, v)(t)+(\delta-1) D_{0^{+}}^{v} e(t) .
\end{aligned}
$$

Therefore, $M(u, v) \geq \delta N(u, v)+(\delta-1) e$. That is, the condition (L5) of Theorem 3.1 is satisfied. Consequently, all the conditions of Theorem 3.1 are satisfied, the conclusions of Theorem 3.2 hold.

By the proof of Theorem 3.2, combining with Theorem 3.1', we can obtain the following result.

Theorem 3.2' Assume that the conditions (H1), (H2), (H3)(a)(b), (H4) and (H5) in Theorem 3.2 are satisfied. Moreover, for fixed $t \in[0,1]$ and $y \in P_{h, e}, \psi(\lambda, x, y)$ are decreasing in $x \in P_{h, e}$ and for fixed $t \in[0,1]$ and $x \in P_{h, e}, \psi(\lambda, x, y)$ are increasing in $y \in P_{h, e}$. In addition, there exists $t_{0} \in(0,1)$ such that

$$
\begin{aligned}
t_{0} h(t) & +\left(t_{0}-1\right) e(t) \\
\leq & \int_{0}^{1} G(t, s)\left(\int_{0}^{1} H(s, \tau) f\left(\tau, h(\tau), D_{0^{+}}^{v} h(\tau)\right) d \tau\right) d s-e(t) \\
\leq & \frac{1}{t_{0}} \psi\left(t_{0}, t_{0}^{-1} h+\left(t_{0}^{-1}-1\right) e, t_{0} h+\left(t_{0}-1\right) e\right) h(t) \\
& +\left[\frac{1}{t_{0}} \psi\left(t_{0}, t_{0}^{-1} h+\left(t_{0}^{-1}-1\right) e, t_{0} h+\left(t_{0}-1\right) e\right)-1\right] e(t) .
\end{aligned}
$$

Then the conclusions of Theorem 3.2 hold.

Lastly, let us give an example to illustrate our main results.

Example 3.1 Consider the following boundary value problem:

$$
\left\{\begin{aligned}
& D_{0^{+}}^{\frac{3}{2}}\left(D_{0^{+}}^{\frac{3}{2}} u\right)(t)= 2 t^{2}+1+\left(u(t)+\frac{15}{\Gamma^{2}\left(\frac{3}{2}\right)}+1\right)^{\frac{1}{3}}+\left(u(t)+\frac{15}{\Gamma^{2}\left(\frac{3}{2}\right)}+1\right)^{\frac{1}{2}} \\
&+\left(D_{0^{+}}^{\frac{1}{8}} u(t)+\frac{15}{\Gamma^{2}\left(\frac{3}{2}\right)}+1\right)^{\frac{-1}{5}} \\
&+\left(\int_{0}^{t}\left(u(s)+\frac{15}{\Gamma^{2}\left(\frac{3}{2}\right)}\right) d s+\frac{15}{\Gamma^{2}\left(\frac{3}{2}\right)}+1\right)^{-1}-10 \\
& u(0)=0, \quad D_{0^{+}}^{\frac{3}{2}} u(0)=0 \\
& D_{0^{+}}^{\frac{1}{4}} u(1)=\frac{1}{10} D_{0^{+}}^{\frac{1}{4}} u\left(\frac{1}{4}\right)+\frac{1}{10} D_{0^{+}}^{\frac{1}{4}} u\left(\frac{1}{2}\right)+\frac{1}{10} D_{0^{+}}^{\frac{1}{4}} u\left(\frac{3}{4}\right) \\
& D_{0^{+}}^{\frac{3}{2}} u(1)=\frac{1}{10} D_{0^{+}}^{\frac{3}{2}} u\left(\frac{1}{4}\right)+\frac{1}{10} D_{0^{+}}^{\frac{3}{2}} u\left(\frac{1}{2}\right)+\frac{1}{10} D_{0^{+}}^{\frac{3}{2}} u\left(\frac{3}{4}\right) .
\end{aligned}\right.
$$

Then the problem (3.6) has a solution.

Proof The problem (1.1) becomes the problem (3.6) when we choose $n=2, \alpha=\frac{3}{2}, \beta=\frac{3}{2}$, $\gamma=\frac{1}{4}, v=\frac{1}{8}, b=10, \eta_{1}=\frac{1}{4}, \eta_{2}=\frac{1}{2}, \eta_{3}=\frac{3}{4}, \xi_{1}=\xi_{2}=\xi_{3}=\frac{1}{10}$, and $\zeta_{1}=\zeta_{2}=\zeta_{3}=\frac{1}{10}$. Then we 
have

$$
\begin{aligned}
& A=1-\sum_{i=1}^{m-2} \xi_{i} \eta_{i}^{\alpha-\gamma-1} \approx 0.7521>0, \\
& B=1-\sum_{i=1}^{m-2} \zeta_{i} \eta_{i}^{\beta-1} \approx 0.7927>0, \\
& F=\frac{1}{B}\left(1+\sum_{i=1}^{m-2} \zeta_{i}\left(1-\eta_{i}^{\beta-1}\right)\right) \approx 1.5571, \\
& D=\frac{1}{A}\left(1+\sum_{i=1}^{m-2} \xi_{i}\left(1-\eta_{i}^{\alpha-\gamma-1}\right)\right) \approx 1.3988 .
\end{aligned}
$$

A direct computation leads to

$$
\begin{aligned}
& \int_{0}^{1} H(s, \tau) d \tau=\int_{0}^{1} H_{1}(s, \tau) d \tau+\int_{0}^{1} H_{2}(s, \tau) d \tau \\
& =\frac{s^{\frac{1}{2}}-s^{\frac{3}{2}}}{\Gamma\left(\frac{5}{2}\right)}+\frac{\frac{3}{10}\left[\frac{1}{4}^{\frac{1}{2}}-\frac{1}{4}^{\frac{3}{2}}+\frac{1}{2}^{\frac{1}{2}}-\frac{1}{2}^{\frac{3}{2}}+\frac{3}{4}^{\frac{1}{2}}-\frac{3}{4}^{\frac{3}{2}}\right] s^{\frac{1}{2}}}{B \Gamma\left(\frac{5}{2}\right)}, \\
& e(t)=10 \int_{0}^{1} G(t, s) \int_{0}^{1} H(s, \tau) d \tau d s \\
& =10 \int_{0}^{1} G(t, s)\left[\frac{s^{\frac{1}{2}}-s^{\frac{3}{2}}}{\Gamma\left(\frac{5}{2}\right)}+\frac{\frac{3}{10}\left[\frac{1}{4}^{\frac{1}{2}}-\frac{1}{4}^{\frac{3}{2}}+\frac{1}{2}^{\frac{1}{2}}-\frac{1}{2}^{\frac{3}{2}}+\frac{3}{4}^{\frac{1}{2}}-\frac{3}{4}^{\frac{3}{2}}\right] s^{\frac{1}{2}}}{B \Gamma\left(\frac{5}{2}\right)}\right] d s \\
& \leq 10 \int_{0}^{1} \frac{D t^{\frac{1}{2}}}{\Gamma\left(\frac{3}{2}\right)}\left[\frac{s^{\frac{1}{2}}-s^{\frac{3}{2}}}{\Gamma\left(\frac{5}{2}\right)}+\frac{\left.\frac{3}{10}_{10} \frac{1}{4}^{\frac{1}{2}}-\frac{1}{4}^{\frac{3}{2}}+\frac{1}{2}^{\frac{1}{2}}-\frac{1}{2}^{\frac{3}{2}}+\frac{3}{4}^{\frac{1}{2}}-\frac{3}{4}^{\frac{3}{2}}\right] s^{\frac{1}{2}}}{B \Gamma\left(\frac{5}{2}\right)}\right] d s \\
& \leq \frac{15}{\Gamma^{2}\left(\frac{3}{2}\right)} t^{\frac{1}{2}}=L t^{\frac{1}{2}}=h(t)
\end{aligned}
$$

and

$$
e^{*} \leq \frac{15}{\Gamma^{2}\left(\frac{3}{2}\right)}, \quad D_{0^{+}}^{\frac{1}{8}} e(t) \leq \frac{15}{\Gamma\left(\frac{3}{2}\right) \Gamma\left(\frac{11}{8}\right)}
$$

Let

$$
\begin{aligned}
& f(t, u, v)=t^{2}+1+\left(u(t)+\frac{15}{\Gamma^{2}\left(\frac{3}{2}\right)}+1\right)^{\frac{1}{2}}+\left(v(t)+\frac{15}{\Gamma^{2}\left(\frac{3}{2}\right)}+1\right)^{-\frac{1}{5}}, \\
& g(t, u, v)=t^{2}+\left(u(t)+\frac{15}{\Gamma^{2}\left(\frac{3}{2}\right)}+1\right)^{\frac{1}{3}}+\left(v(t)+\frac{15}{\Gamma^{2}\left(\frac{3}{2}\right)}+1\right)^{-1}, \\
& (\mathcal{K} u)(t)=\int_{0}^{t}\left(u(s)+\frac{15}{\Gamma^{2}\left(\frac{3}{2}\right)}\right) d s .
\end{aligned}
$$


For $\lambda \in(0,1)$ and $\hat{e} \in\left[0, \frac{15}{\Gamma^{2}\left(\frac{3}{2}\right)}\right]$, we deduce

$$
\begin{aligned}
\mathcal{K}(\lambda u+(\lambda-1) \hat{e}) & =\int_{0}^{t}\left(\lambda u+(\lambda-1) \hat{e}+\frac{15}{\Gamma^{2}\left(\frac{3}{2}\right)}\right) d s \\
& =\lambda \int_{0}^{t} u d s+\int_{0}^{t}(\lambda-1) \hat{e} d s+\int_{0}^{t} \frac{15}{\Gamma^{2}\left(\frac{3}{2}\right)} d s \\
& \geq \lambda(\mathcal{K} u)(t)+(\lambda-1) \hat{e},
\end{aligned}
$$

and $(\mathcal{K} u)$ is increasing in $u$, thus (H5) is satisfied. It is easy to check that $f, g:[0,1] \times$ $\left[-\frac{15}{\Gamma^{2}\left(\frac{3}{2}\right)},+\infty\right) \times\left[-\frac{15}{\Gamma^{2}\left(\frac{3}{2}\right)},+\infty\right) \rightarrow(-\infty,+\infty)$ are continuous, $f(t, u, v), g(t, u, v)$ are both increasing in $u$ and decreasing in $v$ and $g(t, 0, \mathcal{K}(L))=t^{2}+\left(\frac{15}{\Gamma^{2}\left(\frac{3}{2}\right)}+1\right)^{\frac{1}{3}}+\left(\mathcal{K}(L)+\frac{15}{\Gamma^{2}\left(\frac{3}{2}\right)}+1\right)^{-1}>$ 0 . Thus, (H1) and (H2) are satisfied.

For all $\lambda \in(0,1), t \in[0,1], u, v \in P_{h, e}, \rho_{1}, \rho_{3} \in\left[0, \frac{15}{\Gamma^{2}\left(\frac{3}{2}\right)}\right]$ and $\rho_{2} \in\left[0, \frac{15}{\Gamma\left(\frac{3}{2}\right) \Gamma\left(\frac{11}{8}\right)}\right]$, there exists $\psi(\lambda, u, v)=\lambda^{\frac{1}{2}}$ such that

$$
\begin{aligned}
& f\left(t, \lambda u+(\lambda-1) \rho_{1}, \lambda^{-1} D_{0^{+}}^{\frac{1}{8}} \nu+\left(\lambda^{-1}-1\right) \rho_{2}\right) \\
& =t^{2}+1+\left(\lambda u+(\lambda-1) \rho_{1}+\frac{15}{\Gamma^{2}\left(\frac{3}{2}\right)}+1\right)^{\frac{1}{2}} \\
& +\left(\lambda^{-1} D_{0^{+}}^{\frac{1}{8}} \nu+\left(\lambda^{-1}-1\right) \rho_{2}+\frac{15}{\Gamma^{2}\left(\frac{3}{2}\right)}+1\right)^{-\frac{1}{5}} \\
& =t^{2}+1+\lambda^{\frac{1}{2}}\left(u+\left(1-\lambda^{-1}\right) \rho_{1}+\lambda^{-1} \frac{15}{\Gamma^{2}\left(\frac{3}{2}\right)}+\lambda^{-1}\right)^{\frac{1}{2}} \\
& +\lambda^{\frac{1}{5}}\left(D_{0^{+}}^{\frac{1}{8}} \nu+(1-\lambda) \rho_{2}+\lambda \frac{15}{\Gamma^{2}\left(\frac{3}{2}\right)}+\lambda\right)^{-\frac{1}{5}} \\
& \geq t^{2}+1+\lambda^{\frac{1}{2}}\left(u+\left(1-\lambda^{-1}\right) \frac{15}{\Gamma^{2}\left(\frac{3}{2}\right)}+\lambda^{-1} \frac{15}{\Gamma^{2}\left(\frac{3}{2}\right)}+\lambda^{-1}\right)^{\frac{1}{2}} \\
& +\lambda^{\frac{1}{5}}\left(D_{0^{+}}^{\frac{1}{8}} \nu+(1-\lambda) \frac{15}{\Gamma\left(\frac{3}{2}\right) \Gamma\left(\frac{11}{8}\right)}+\lambda \frac{15}{\Gamma^{2}\left(\frac{3}{2}\right)}+\lambda\right)^{-\frac{1}{5}} \\
& \geq t^{2}+1+\lambda^{\frac{1}{2}}\left(u+\frac{15}{\Gamma^{2}\left(\frac{3}{2}\right)}+1\right)^{\frac{1}{2}} \\
& +\lambda^{\frac{1}{5}}\left(D_{0^{+}}^{\frac{1}{8}} \nu+(1-\lambda) \frac{15}{\Gamma^{2}\left(\frac{3}{2}\right)}+\lambda \frac{15}{\Gamma^{2}\left(\frac{3}{2}\right)}+\lambda\right)^{-\frac{1}{5}} \\
& \geq t^{2}+1+\lambda^{\frac{1}{2}}\left(u+\frac{15}{\Gamma^{2}\left(\frac{3}{2}\right)}+1\right)^{\frac{1}{2}}+\lambda^{\frac{1}{5}}\left(D_{0^{+}}^{\frac{1}{8}} \nu+\frac{15}{\Gamma^{2}\left(\frac{3}{2}\right)}+1\right)^{-\frac{1}{5}} \\
& \geq \lambda^{\frac{1}{2}}\left[t^{2}+1+\left(u+\frac{15}{\Gamma^{2}\left(\frac{3}{2}\right)}+1\right)^{\frac{1}{2}}+\left(D_{0^{+}}^{\frac{1}{8}} v+\frac{15}{\Gamma^{2}\left(\frac{3}{2}\right)}+1\right)^{-\frac{1}{5}}\right] \\
& =\psi(\lambda, u, v) f\left(t, u, D_{0^{+}}^{\frac{1}{8}} v\right) \text {. }
\end{aligned}
$$


Moreover, we deduce

$$
\begin{aligned}
g\left(t, \lambda u+(\lambda-1) \rho_{3}, \lambda^{-1} v+\left(\lambda^{-1}-1\right) \rho_{3}\right) & \\
= & t^{2}+\left(\lambda u+(\lambda-1) \rho_{3}+\frac{15}{\Gamma^{2}\left(\frac{3}{2}\right)}+1\right)^{\frac{1}{3}}+\left(\lambda^{-1} v+\left(\lambda^{-1}-1\right) \rho_{3}+\frac{15}{\Gamma^{2}\left(\frac{3}{2}\right)}+1\right)^{-1} \\
= & t^{2}+\lambda^{\frac{1}{3}}\left(u+\left(1-\lambda^{-1}\right) \rho_{3}+\frac{15 \lambda^{-1}}{\Gamma^{2}\left(\frac{3}{2}\right)}+\lambda^{-1}\right)^{\frac{1}{3}}+\lambda\left(v+(1-\lambda) \rho_{3}+\frac{15 \lambda}{\Gamma^{2}\left(\frac{3}{2}\right)}+\lambda\right)^{-1} \\
\geq & \lambda t^{2}+\lambda\left(u+\left(1-\lambda^{-1}\right) \frac{15}{\Gamma^{2}\left(\frac{3}{2}\right)}+\frac{15 \lambda^{-1}}{\Gamma^{2}\left(\frac{3}{2}\right)}+\lambda^{-1}\right)^{\frac{1}{3}} \\
& +\lambda\left(v+(1-\lambda) \frac{15}{\Gamma^{2}\left(\frac{3}{2}\right)}+\frac{15 \lambda}{\Gamma^{2}\left(\frac{3}{2}\right)}+\lambda\right)^{-1} \\
= & \lambda t^{2}+\lambda\left(u+\frac{15}{\Gamma^{2}\left(\frac{3}{2}\right)}+\lambda^{-1}\right)^{\frac{1}{3}}+\lambda\left(v+\frac{15}{\Gamma^{2}\left(\frac{3}{2}\right)}+\lambda\right)^{-1} \\
\geq & \lambda\left[t^{2}+\left(u+\frac{15}{\Gamma^{2}\left(\frac{3}{2}\right)}+1\right)^{\frac{1}{3}}+\left(v+\frac{15}{\Gamma^{2}\left(\frac{3}{2}\right)}+1\right)^{-1}\right] \\
= & \lambda g(t, u, v) .
\end{aligned}
$$

Thus, (H3) is satisfied. Furthermore, for $u, v \in P_{h, e}$, we get

$$
\begin{aligned}
f(t, u, v) & =t^{2}+1+\left(u(t)+\frac{15}{\Gamma^{2}\left(\frac{3}{2}\right)}+1\right)^{\frac{1}{2}}+\left(v(t)+\frac{15}{\Gamma^{2}\left(\frac{3}{2}\right)}+1\right)^{-\frac{1}{5}} \\
& \geq t^{2}+1+\left(u(t)+\frac{15}{\Gamma^{2}\left(\frac{3}{2}\right)}+1\right)^{\frac{1}{3}}+\left(v(t)+\frac{15}{\Gamma^{2}\left(\frac{3}{2}\right)}+1\right)^{-1} \\
& \geq t^{2}+\left(u(t)+\frac{15}{\Gamma^{2}\left(\frac{3}{2}\right)}+1\right)^{\frac{1}{3}}+\left(\frac{15}{\Gamma^{2}\left(\frac{3}{2}\right)}+1\right)^{-1}=g(t, u, 0),
\end{aligned}
$$

let $\delta=1$, we have $f(t, u, v) \geq \delta g(t, u, 0)$. Thus (H4) is satisfied.

By Lemmas 2.4 and 2.5, we have

$$
\begin{aligned}
& \int_{0}^{1} G(t, s)\left(\int_{0}^{1} H(s, \tau) f\left(\tau, h(\tau), D_{0^{+}}^{v} h(\tau)\right) d \tau\right) d s \\
& \quad \leq \int_{0}^{1} G(t, s)\left(\int_{0}^{1} H(s, \tau)\left(4+h(\tau)+\frac{15}{\Gamma^{2}\left(\frac{3}{2}\right)}\right) d \tau\right) d s \\
& \quad=\int_{0}^{1} G(t, s)\left(\int_{0}^{1} H(s, \tau) h(\tau) d \tau\right) d s+\left[\frac{15}{\Gamma^{2}\left(\frac{3}{2}\right)}+4\right] \frac{e(t)}{10} \\
& \leq \int_{0}^{1} \frac{D t^{\frac{1}{2}}}{\Gamma\left(\frac{3}{2}\right)}\left(\int_{0}^{1} \frac{15 F^{\frac{1}{2}} \tau^{\frac{1}{2}}}{\Gamma^{3}\left(\frac{3}{2}\right)} d \tau\right) d s+\left[\frac{15}{\Gamma^{2}\left(\frac{3}{2}\right)}+4\right] \frac{e(t)}{10} \\
& =\frac{4 D F}{9 \Gamma^{2}\left(\frac{3}{2}\right)} h(t)+\left[\frac{15}{\Gamma^{2}\left(\frac{3}{2}\right)}+4\right] \frac{e(t)}{10} \\
& =1.2325 h(t)+2.3099 e(t)
\end{aligned}
$$


and

$$
\begin{aligned}
& \int_{0}^{1} G(t, s)\left(\int_{0}^{1} H(s, \tau) f\left(\tau, h(\tau), D_{0^{+}}^{v} h(\tau)\right) d \tau\right) d s \\
& \quad \geq \int_{0}^{1} \frac{c(s) t^{\frac{1}{2}}}{\Gamma\left(\frac{3}{2}\right)}\left(\int_{s}^{1} \frac{1}{\Gamma\left(\frac{3}{2}\right)} s^{\frac{1}{2}}(1-\tau)^{\frac{1}{2}} \tau^{\frac{1}{4}} d \tau\right) d s+\frac{1}{10} e(t) \\
& \quad \geq \frac{4 t^{\frac{1}{2}}}{5 \Gamma^{2}\left(\frac{3}{2}\right)} \int_{0}^{1} c(s) s^{\frac{1}{2}}\left(1-s^{\frac{5}{4}}\right) d s+\frac{1}{10} e(t) \\
& \quad \geq \frac{4 t^{\frac{1}{2}}}{5 \Gamma^{2}\left(\frac{3}{2}\right)} \int_{\frac{1}{2}}^{1} \frac{1}{10}\left(\frac{1}{2}\right)^{\frac{1}{4}}(1-s)^{\frac{1}{4}} s^{\frac{1}{2}}\left(1-s^{\frac{5}{4}}\right) d s+\frac{1}{10} e(t) \\
& \geq 0.0001 h(t)+0.1 e(t) .
\end{aligned}
$$

Choose $t_{0}=10^{-8}$, we deduce that the condition (H6) is satisfied. Therefore, all the assumptions of Theorem 3.2 are satisfied. We can construct the following iteration sequences:

$$
\begin{aligned}
\omega_{n}(t)= & \int_{0}^{1} G(t, s)\left(\int _ { 0 } ^ { 1 } H ( s , \tau ) \left(\tau^{2}+\left(\omega_{n-1}(\tau)+\frac{15}{\Gamma^{2}\left(\frac{3}{2}\right)}+1\right)^{\frac{1}{3}}\right.\right. \\
& \left.\left.+\left(\int_{0}^{\tau}\left(\sigma_{n-1}(x)+\frac{15}{\Gamma^{2}\left(\frac{3}{2}\right)}\right) d x+\frac{15}{\Gamma^{2}\left(\frac{3}{2}\right)}+1\right)^{-1}\right) d \tau\right) d s \\
& +\int_{0}^{1} G(t, s)\left(\int _ { 0 } ^ { 1 } H ( s , \tau ) \left(\tau^{2}+1+\left(\omega_{n-1}(\tau)+\frac{15}{\Gamma^{2}\left(\frac{3}{2}\right)}+1\right)^{\frac{1}{2}}\right.\right. \\
& \left.\left.+\left(D_{0^{+}}^{v} \sigma_{n-1}(\tau)+\frac{15}{\Gamma^{2}\left(\frac{3}{2}\right)}+1\right)^{-\frac{1}{5}}\right) d \tau\right) d s-e(t), \quad n=1,2, \ldots
\end{aligned}
$$

and

$$
\begin{aligned}
\sigma_{n}(t)= & \int_{0}^{1} G(t, s)\left(\int _ { 0 } ^ { 1 } H ( s , \tau ) \left(\tau^{2}+\left(\sigma_{n-1}(\tau)+\frac{15}{\Gamma^{2}\left(\frac{3}{2}\right)}+1\right)^{\frac{1}{3}}\right.\right. \\
& \left.\left.+\left(\int_{0}^{\tau}\left(\omega_{n-1}(x)+\frac{15}{\Gamma^{2}\left(\frac{3}{2}\right)}\right) d x+\frac{15}{\Gamma^{2}\left(\frac{3}{2}\right)}+1\right)^{-1}\right) d \tau\right) d s \\
& +\int_{0}^{1} G(t, s)\left(\int _ { 0 } ^ { 1 } H ( s , \tau ) \left(\tau^{2}+1+\left(\sigma_{n-1}(\tau)+\frac{15}{\Gamma^{2}\left(\frac{3}{2}\right)}+1\right)^{\frac{1}{2}}\right.\right. \\
& \left.\left.\left.+\left(D_{0^{+}}^{v} \omega_{n-1}\right)(\tau)+\frac{15}{\Gamma^{2}\left(\frac{3}{2}\right)}+1\right)^{-\frac{1}{5}}\right) d \tau\right) d s-e(t), \quad n=1,2, \ldots,
\end{aligned}
$$

for any initial values $\omega_{0}, \sigma_{0} \in P_{h, e}$, we have $\omega_{n} \rightarrow u^{*}$ and $\sigma_{n} \rightarrow u^{*}$ as $n \rightarrow \infty$.

\section{Conclusions}

In this paper, we obtain two new mixed monotone fixed point theorems. By using our abstract results, we establish the existence and uniqueness theorems of the solution for a fractional $m$-point boundary value problem, which generalizes the well-known elastic beam equation. Furthermore, two iterative sequences to approximate the unique solution are also given. 


\section{Acknowledgements}

Not applicable.

\section{Funding}

This project is supported by the Programs for the Cultivation of Young Scientific Research Personnel of Higher Education Institutions in Shanxi Province, the Scientific and Technological Innovation Programs of Higher Education Institutions in Shanxi (201802085), the Innovative Research Team of North University of China (TD201901), and the Natural Science Foundation of Shanxi Province(201801D121027).

\section{Availability of data and materials}

Data sharing not applicable to this article as no data sets were generated or analyzed during the current study.

\section{Competing interests}

The authors declare that they have no competing interests.

\section{Authors' contributions}

All authors contributed equally to this work. All authors read and approved the final manuscript.

\section{Publisher's Note}

Springer Nature remains neutral with regard to jurisdictional claims in published maps and institutional affiliations.

\section{Received: 8 July 2020 Accepted: 7 February 2021 Published online: 19 February 2021}

\section{References}

1. Lv, Z.W.: Positive solutions of $m$-point boundary value problems for fractional differential equations. Adv. Differ. Equ. 2011, 571804 (2011)

2. LV, Z.W.: Existence results for $m$-point boundary value problems of nonlinear fractional differential equations with p-Laplacian operator. Adv. Differ. Equ. 2014, 69 (2014)

3. Jong, K.S.: Existence and uniqueness of positive solutions of a kind of multi-point boundary value problems for nonlinear fractional differential equations with $p$-Laplacian operator. Mediterr. J. Math. 15, 129 (2018)

4. Jong, K.S., Choi, H., Ri, Y.: Existence of positive solutions of a class of multi-point boundary value problems for p-Laplacian fractional differential equations with singular source terms. Commun. Nonlinear Sci. Numer. Simul. 72, 272-281 (2019)

5. Li, C.F., Luo, X.N., Zhou, Y.: Existence of positive solutions of the boundary value problem for nonlinear fractional differential equations. Comput. Math. Appl. 59, 1363-1375 (2010)

6. Wang, H., Zhang, L.L., Wang, X.Q.: New unique existence criteria for higher-order nonlinear singular fractional differential equations. Nonlinear Anal., Model. Control 24,95-120 (2019)

7. Liang, S., Zhang, J.: Existence and uniqueness of strictly nondecreasing and positive solution for a fractional three-point boundary value problem. Comput. Math. Appl. 62, 1333-1340 (2011)

8. Jleli, M., Samet, B.: Existence of positive solutions to an arbitrary order fractional differential equation via a mixed monotone operator method. Nonlinear Anal., Model. Control 20, 367-376 (2015)

9. Wang, T:: The study on mixed monotone operator theories and solutions for several differential equations. Doctoral dissertation, Qufu Normal University, Qufu (2018)

10. Zhang, L.L., Tian, H.M.: Existence and uniqueness of positive solutions for a class of nonlinear fractional differential equations. Adv. Differ. Equ. 2017, 114 (2017)

11. Ji, Y.D., Guo, Y.P., Qiu, J.Q., Yang, L.Y.: Existence of positive solutions for a boundary value problem of nonlinear fractional differential equations. Adv. Differ. Equ. 2015, 13 (2015)

12. Goodrich, C.S.: Existence of a positive solution to a class of fractional differential equations. Appl. Math. Lett. 23, 1050-1055 (2010)

13. Bai, C.Z.: Triple positive solutions for a boundary value problem of nonlinear fractional differential equation. Electron. J. Qual. Theory Differ. Equ. 2008, 24 (2008)

14. Zhang, S.: Positive solutions to singular boundary value problem for nonlinear fractional differential equation. Comput. Math. Appl. 59, 1300-1309 (2010)

15. Yue, Z.Z., Zou, Y.M.: New uniqueness results for fractional differential equation with dependence on the first order derivative. Adv. Differ. Equ. 2019, 38 (2019)

16. Zhang, $X$. ., Liu, L., Wu, Y.: The eigenvalue problem for a singular higher order fractional differential equation involving fractional derivatives. Comput. Math. Appl. 218, 8526-8536 (2012)

17. Li, Y.H., Qi, A.B.: Positive solutions for multi-point boundary value problems of fractional differential equations with p-Laplacian. Math. Methods Appl. Sci. 39, 1425-1434 (2016)

18. Tan, J.J., Li, M.: Solutions of fractional differential equations with $p$-Laplacian operator in Banach spaces. Bound. Value Probl. 2018, 15 (2018)

19. Wang, J.H., Xiang, H.J.: Upper and lower solutions method for a class of singular fractional boundary value problems with p-Laplacian operator. Abstr. Appl. Anal. 2010, 971824 (2010)

20. Wang, J., Xiang, H., Liu, Z.: Positive solutions for three-point boundary value problems of nonlinear fractional differential equations with p-Laplacian. Far East J. Appl. Math. 37, 33-47 (2009)

21. Sang, Y.B., Ren, Y.: Nonlinear sum operator equations and applications to elastic beam equation and fractional differential equation. Bound. Value Probl. 2019, 49 (2019)

22. Zhai, C.B., Wang, L.: $\varphi$-(h,e) concave operators and applications. J. Math. Anal. Appl. 454, 571-584 (2017)

23. Ahmad, B., Ntouyas, S., Alsaedi, A.: On a coupled system of fractional differential equations with coupled nonlocal and integral boundary conditions. Chaos Solitons Fractals 83, 234-241 (2016) 
24. Zhai, C., Xu, L.: Properties of positive solutions to a class of four-point boundary value problem of Caputo fractional differential equations with a parameter. Commun. Nonlinear Sci. Numer. Simul. 19, 2820-2827 (2014)

25. Jiang, J., O'Regan, D., Xu, J., Fu, Z.: Positive solutions for a system of nonlinear Hadamard fractional differential equations involving coupled integral boundary conditions. J. Inequal. Appl. 2019, 204 (2019)

26. Ren, J., Zhai, C.: Nonlocal q-fractional boundary value problem with Stieltjes integral conditions. Nonlinear Anal., Model. Control 24(4), 582-602 (2019)

27. Wang, G., Pei, K., Chen, Y.: Stability analysis of nonlinear Hadamard fractional differential system. J. Franklin Inst. 356, 6538-6546 (2019)

28. Zhai, C., Wang, W., Li, H.: A uniqueness method to a new Hadamard fractional differential system with four-point boundary conditions. J. Inequal. Appl. 2018, 207 (2018)

29. Zhai, C., Ren, J.: The unique solution for a fractional q-difference equation with three-point boundary conditions. Indag. Math. 29, 948-961 (2018)

30. Guo, D., Lakshmikantham, V.: Nonlinear Problems in Abstract Cones. Academic Press, Boston (1988)

31. Guo, D.: Partial Order Methods in Nonlinear Analysis. Shandong Science and Technology Press, Jinan (2000) (in Chinese)

32. Podlubny, l.: Fractional Differential Equations. Mathematics in Science and Engineering. Academic Press, New York (1999)

33. Kilbas, A., Srivastava, H., Trujillo, J.J.: Theory and Applications of Fractional Differential Equations. North-Holland Mathematics Studies. Elsevier, Amsterdam (2006)

34. Bai, Z., LV, H.: Positive solutions for boundary value problem of nonlinear fractional differential equation. J. Math. Anal. Appl. 311, 495-505 (2005)

\section{Submit your manuscript to a SpringerOpen ${ }^{\circ}$ journal and benefit from:}

- Convenient online submission

- Rigorous peer review

- Open access: articles freely available online

- High visibility within the field

- Retaining the copyright to your article

Submit your next manuscript at $\boldsymbol{~ s p r i n g e r o p e n . c o m ~}$ 